\title{
DISTANCE DETERMINATION TO 12 TYPE II SUPERNOVAE USING THE EXPANDING PHOTOSPHERE METHOD
}

\author{
M. I. Jones ${ }^{1}$, Mario Hamuy ${ }^{1}$, P. Lira ${ }^{1}$, J. Maza ${ }^{1}$, A. Clocchiatti ${ }^{2}$, M. Phillips ${ }^{3}$, N. Morrell ${ }^{3}$, M. Roth ${ }^{3}$, N. B. SuntzefF ${ }^{4}$, \\ T. MAtheson ${ }^{5}$, A. V. Filippenko ${ }^{6}$, R. J. Foley ${ }^{6}$, And D. C. LeOnARd ${ }^{7}$ \\ ${ }^{1}$ Departamento de Astronomía, Universidad de Chile, Chile \\ ${ }^{2}$ Departamento de Astronomía y Astrofísica, Pontificia Universidad Católica de Chile, Chile \\ ${ }^{3}$ Las Campanas Observatory, Carnegie Observatories, La Serena, Chile \\ ${ }^{4}$ Texas A\&M University, Physics Department, College Station, TX, USA \\ ${ }^{5}$ National Optical Astronomy Observatory, 950 North Cherry Avenue, Tucson, AZ 85719-4933, USA \\ ${ }^{6}$ Department of Astronomy, University of California, Berkeley, CA 94720-3411, USA \\ ${ }^{7}$ Department of Astronomy, San Diego State University, San Diego, CA 92182, USA \\ Received 2008 September 1; accepted 2009 February 24; published 2009 April 22
}

\begin{abstract}
We use early-time photometry and spectroscopy of 12 Type II plateau supernovae (SNe IIP) to derive their distances using the expanding photosphere method (EPM). We perform this study using two sets of Type II supernova (SN II) atmosphere models, three filter subsets $(\{B V\},\{B V I\}$, and $\{V I\})$, and two methods for the host-galaxy extinction, which leads to 12 Hubble diagrams. We find that systematic differences in the atmosphere models lead to $\sim 50 \%$ differences in the EPM distances and to a value of $H_{0}$ between 52 and 101 $\mathrm{km} \mathrm{s}^{-1} \mathrm{Mpc}^{-1}$. Using the $\{V I\}$ filter subset we obtain the lowest dispersion in the Hubble diagram, $\sigma_{\mu}=0.32$ mag. We also apply the EPM analysis to the well observed SN IIP $1999 \mathrm{em}$. With the $\{V I\}$ filter subset we derive a distance ranging from $9.3 \pm 0.5 \mathrm{Mpc}$ to $13.9 \pm 1.4 \mathrm{Mpc}$ depending on the atmosphere model employed.
\end{abstract}

Key words: distance scale - supernovae: general

\section{INTRODUCTION}

Type II supernovae (SNe II) are understood as the result of the final gravitational collapse of massive stars $\left(M>8 M_{\odot}\right)$ that, at the moment of the explosion, have most of their hydrogen envelope intact. The energy released in the explosion is typically $\sim 10^{53}$ erg (mainly radiated in the form of neutrinos), and the luminosity of the supernova (SN) during the first few months after explosion can be comparable to the total luminosity of its host galaxy. These objects have been classified based on their light curves into Type IIP (plateau) and Type IIL (linear; e.g., Patat et al. 1994). The former present a nearly constant optical luminosity during the photospheric phase $(\sim 100$ days after explosion), while the latter show a slow decline in luminosity during that phase. However, there are some SN II events, such as the SN 1987A, that show peculiar photometric properties. Also, studies of SN II spectra have revealed the existence of a subclass, characterized by the presence of narrow lines, called SNe IIn (Schlegel 1990; Filippenko 1991b, 1991a), which are most likely originated from the interaction of the SN ejecta with pre-existing circumstellar material; see Filippenko (1997) for a general review of SN spectra.

Due to their high intrinsic luminosities, SNe II have great potential as extragalactic distance indicators. To date, several methods have been proposed to derive distances to SNe II, but two are the most commonly used: the expanding photosphere method (EPM; Kirshner \& Kwan 1974) and the standardized candle method (SCM; Hamuy \& Pinto 2002). The former is a geometrical technique that relates the photospheric radius and the angular radius of a SN in order to derive its distance, and has been applied to several $\mathrm{SNe}$ to derive the Hubble constant (e.g., Schmidt et al. 1992). The EPM is independent of the extragalactic distance ladder, and therefore does not need any external calibration. The SCM is based on the observed relation between expansion velocity and luminosity of SNe IIP. Recently, this method has been applied to a sample of high-redshift SNe (Nugent et al. 2006). Other methods have also been used to determine distances to SNe II, such as the spectral-fitting expanding atmosphere method (SEAM; Baron et al. 2004) and the plateau-tail relation proposed by Nadyozhin (2003).

In this work we apply the EPM using early spectroscopy and photometry of 12 SNe IIP in order to derive their distances. We apply the method using two sets of SN II atmosphere models (Eastman et al. 1996; Dessart \& Hillier 2005a), three filter subsets $(\{B V\},\{B V I\}$, and $\{V I\})$, and two methods for the host-galaxy extinction. The different combinations lead to 12 Hubble diagrams. Section 2 of this paper describes the photometric and spectroscopic observations. In Section 3, the EPM is presented, and we apply it to $12 \mathrm{SNe}$ IIP. The results are discussed in Section 4. We compare our EPM distances with results from other methods and with previous EPM analyses. We also discuss the error analysis and the effect of reddening on the EPM distances. We show 12 Hubble diagrams and the corresponding Hubble constants, and we propose an external calibration for the EPM. Finally, we summarize our conclusions in Section 5.

\section{OBSERVATIONS}

In this work we use photometry and spectroscopy from four SN follow-up programs: the Cerro Tololo supernova program (1986-1996), the Calán/Tololo supernova survey (CT; 19901993), the Supernova Optical and Infrared Survey (SOIRS; 1999-2000), and the Carnegie Type II Supernova Program (CATS; 2002-2003). During these programs optical (and some IR) photometry and spectroscopy were obtained for nearly 100 SNe, 51 of which belong to the Type II class. All of the optical data have already been reduced and will soon be published (M. Hamuy 2009, in preparation). We also complemented our data set with some spectra from various coauthors of this paper. 

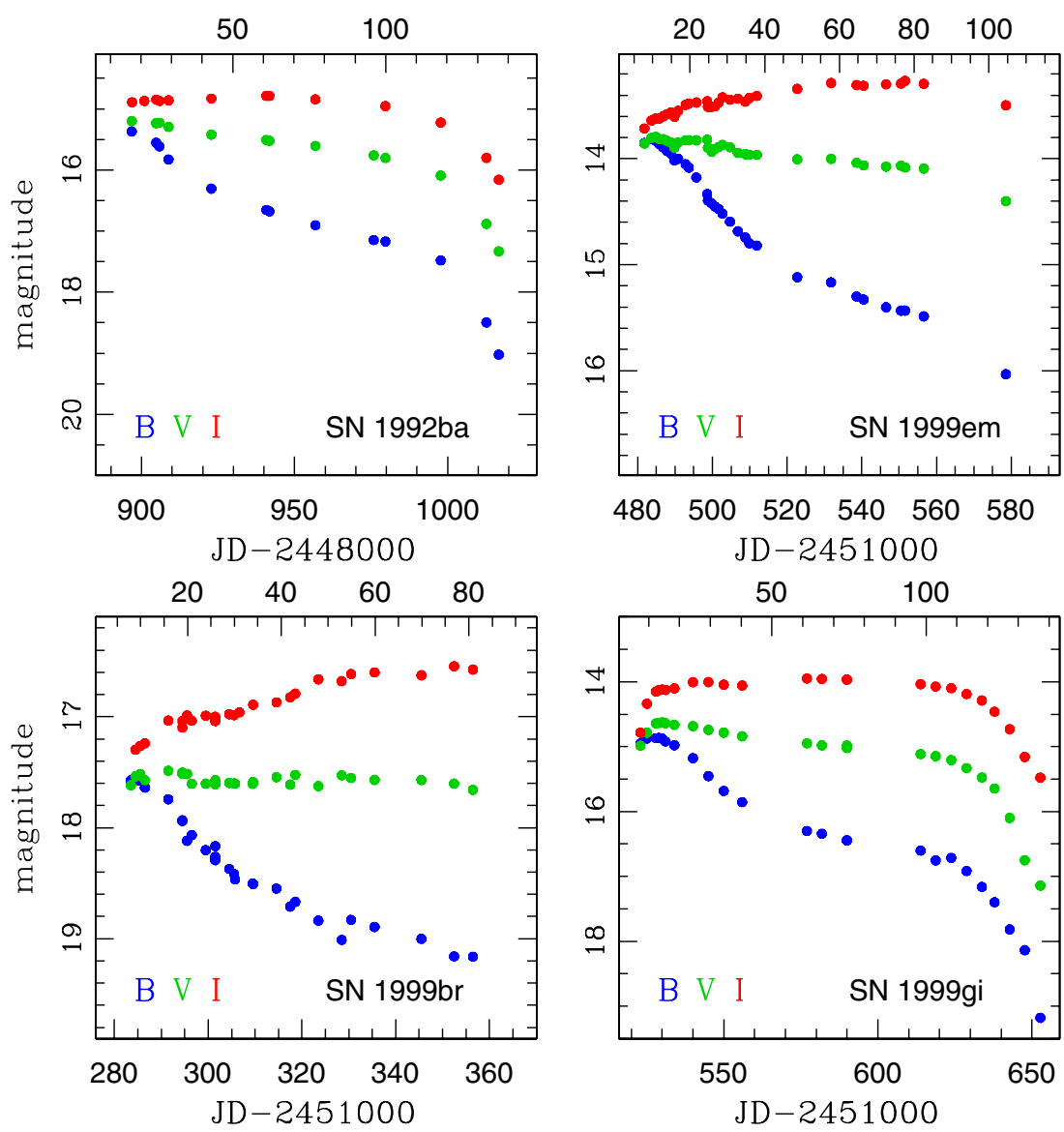

Figure 1. Optical light curves of four SNe during the first $\sim 120$ days of their evolution. The top axis of each panel gives the phase in days since the EPM explosion time, derived using the D05 models (see Table 5).

(A color version of this figure is available in the online journal.)

\subsection{Photometry}

Direct images of $\mathrm{SNe}$ were obtained with telescopes from four different observatories: the Cerro Tololo Inter-American Observatory (CTIO), the Las Campanas Observatory (LCO), the European Southern Observatory (ESO) in La Silla, and the Steward Observatory (S0). Several telescopes and instruments were used to obtain the photometry, which is listed in Jones (2008). In all cases CCD detectors and standard JohnsonKron-Cousins UBVRIZ filters (Johnson et al. 1966; Cousins 1971) were employed. For a small subset of SNe observations in the $J H K$ filters were also obtained. The data reduction was performed using IRAF ${ }^{8}$ according to the procedure described by M. Hamuy (2009, in preparation). The error in the photometry ranges between 0.01 and $0.06 \mathrm{mag}$, with a typical value of $0.02 \mathrm{mag}$.

The optical light curves of all the $\mathrm{SNe}$ used in this work are shown in Figures 1-3, clearly revealing the plateau nature of all these events.

\subsection{Spectroscopy}

Low-resolution $(R \sim 1000)$ optical spectra (wavelength range $\sim 3200-10,000 \AA$ ) were taken for each $\mathrm{SN}$ at various epochs using telescopes and instruments from four different observatories. Jones (2008) lists all the telescopes and instruments

8 IRAF is distributed by the National Optical Astronomy Observatory, which is operated by the Association of Universities for Research in Astronomy, Inc., under cooperative agreement with the National Science Foundation. used for the spectroscopy. Most of the spectra were obtained with the slit along the parallactic angle (Filippenko 1982). The wavelength calibration was performed using comparison-lamp spectra taken at the position of each SN. The flux calibration was done via observations of flux-standard stars (Hamuy et al. 1992, 1994). For more details on the observational procedures see M. Hamuy (2009, in preparation).

The spectra were shifted to the rest frame using the heliocentric redshifts given in Table 1 in order to measure the SN ejecta velocities. In seven cases we were able to measure the redshifts from narrow emission lines of $\mathrm{H}$ II regions at the $\mathrm{SN}$ position (see Table 1). Also, in one case (SN 1999em) we adopted the value from Leonard et al. (2002b) which corresponds to the redshift measured at the $\mathrm{SN}$ position. In four cases we were unable to extract this information from our data, and we had to rely on redshifts of the host-galaxy nuclei; this does not take into account the rotation velocities of the host galaxies, which are typically $v \sim 200 \mathrm{~km} \mathrm{~s}^{-1}$.

\subsection{Sample of Supernovae Used in this Work}

Fifty-one SNe II were observed in the surveys described above. We cut this sample according to the EPM requirements, which are (1) the optical SN light curve ( $V$ and $I$ bands) must show a nearly constant luminosity during the photospheric phase, i.e., the SN must belong to the SN IIP class ( see Figures 13); (2) the SN must have early-time photometry; and (3) the SN must have at least three early spectroscopic observations. The necessity for all of these requirements, discussed in Section 3.6, 

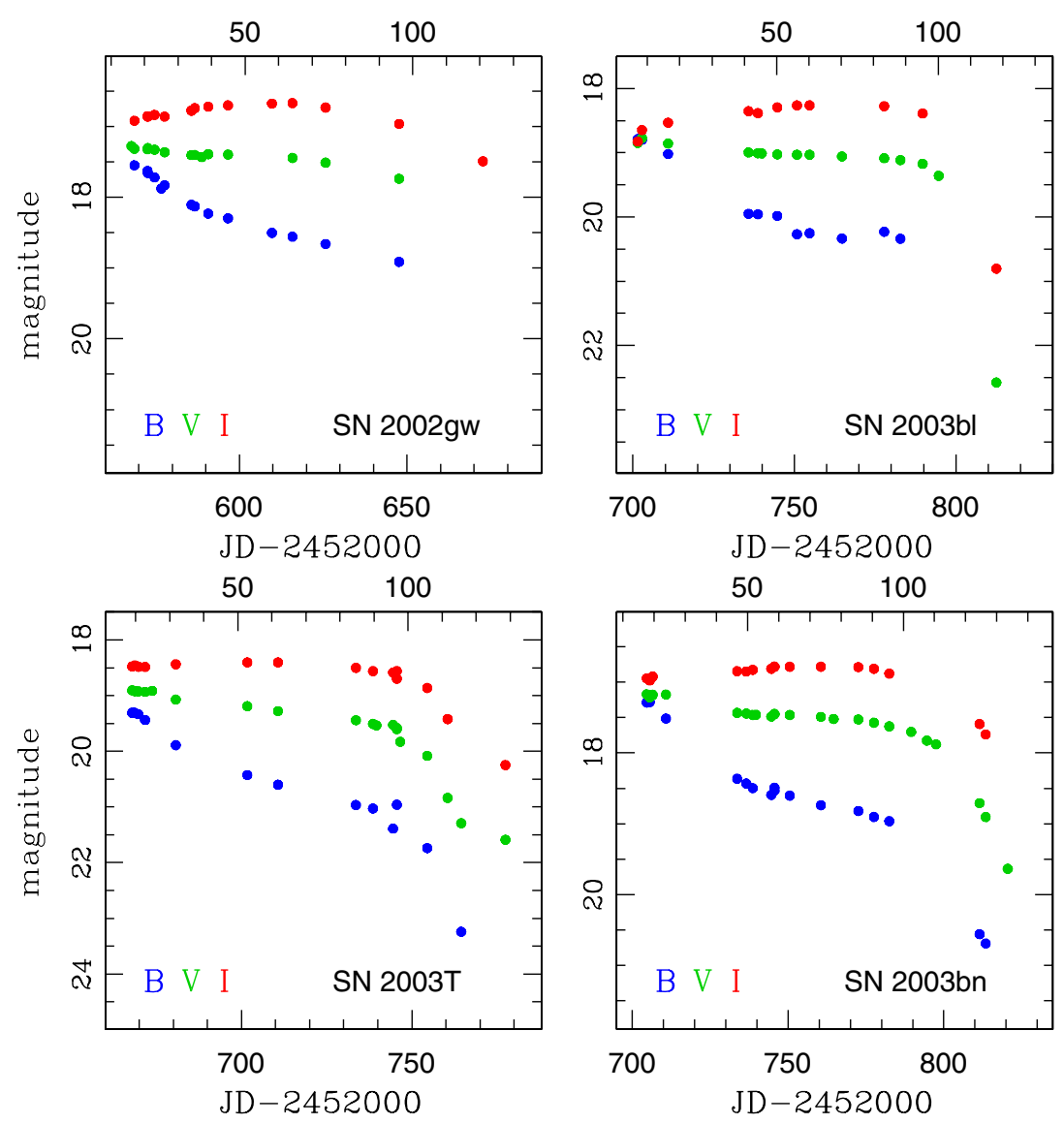

Figure 2. Optical light curves of four SNe during the first $\sim 120$ days of their evolution. The top axis of each panel gives the phase in days since the EPM explosion time, derived using the D05 models (see Table 5).

(A color version of this figure is available in the online journal.)

reduced the sample to only $11 \mathrm{SNe}$. We also added the SN IIP 1999gi to our sample, which has extensive photometry and spectroscopy published by Leonard et al. (2002a).

\section{THE EXPANDING PHOTOSPHERE METHOD}

\subsection{Basic Principles}

The EPM is a geometrical technique that relates an angular size and a physical size of a $\mathrm{SN}$, in order to derive its distance. Although the angular radius $\theta$ of a SN cannot be resolved spatially with current optical instrumentation, it can be derived assuming a spherically symmetric expanding photosphere (a reasonable assumption for SNe IIP at early times, as discussed by Leonard et al. 2001) that radiates as a blackbody "diluted" by a factor $\zeta^{2}$. Specifically,

$$
\theta=\frac{R}{D}=\sqrt{\frac{(1+z) f_{\lambda}}{\pi \zeta_{\lambda^{\prime}}^{2} B_{\lambda^{\prime}}(T) 10^{-0.4\left[A(\lambda)+A^{\prime}\left(\lambda^{\prime}\right)\right]}},}
$$

where $R$ is the photospheric radius, $D$ is the distance to the $\mathrm{SN}$, $f_{\lambda}$ is the observed flux density, $\lambda$ is the observed wavelength, $B_{\lambda^{\prime}}$ is the Planck function in the SN rest frame, $T$ is the color temperature, $\lambda^{\prime}=\lambda /(1+z)$ is the corresponding wavelength in the SN rest frame, $A(\lambda)$ is the foreground dust extinction, and $A^{\prime}\left(\lambda^{\prime}\right)$ is the host-galaxy extinction. The factor $\zeta_{\lambda^{\prime}}$ (known as "distance correction factor" or "dilution factor") accounts for the fact that a SN does not radiate as a perfect blackbody; there is flux dilution caused by gray electron scattering which makes the photosphere (defined as the region of total optical depth $\tau=2 / 3$ ) form in a layer above the thermalization surface. Also, the dilution factor accounts for line blanketing in the $\mathrm{SN}$ atmosphere. Since electron scattering is the main source of continuum opacity, the total opacity is essentially gray, and the photospheric angular radius is independent of wavelength in the optical and near-infrared (Eastman et al. 1996), which explains why $R$ and $\theta$ do not carry a wavelength subscript.

Because the gravitational binding energy $\left(U \sim 10^{49} \mathrm{erg}\right)$ of a SN progenitor is far less than the expansion kinetic energy ( $E \sim 10^{51} \mathrm{erg}$ ) of the ejecta, it is reasonable to assume free expansion. This assumption is supported by hydrodynamical models which show that the different layers of the ejecta reach $\sim 95 \%$ of their terminal velocities $\sim 1$ day after the explosion. During this brief period there is a transition from an acceleration phase due to the SN explosion, to homologous expansion (Utrobin 2007; Bersten et al. 2009, in preparation). Due to the high expansion velocities $\left(\sim 10,000 \mathrm{~km} \mathrm{~s}^{-1}\right)$, the initial radius (typically $R_{0} \sim 10^{13} \mathrm{~cm}$ for a red supergiant) can be neglected after $\sim 1$ day from explosion; hence after that period the physical radius of the $\mathrm{SN}$ can be approximated by

$$
R \approx \frac{v\left(t-t_{0}\right)}{1+z}
$$

where $v$ is the photospheric velocity and $t_{0}$ is the explosion date. Combining (1) and (2) we obtain

$$
\frac{\theta_{i}}{v_{i}} \approx \frac{\left(t_{i}-t_{0}\right)}{(1+z) D}
$$



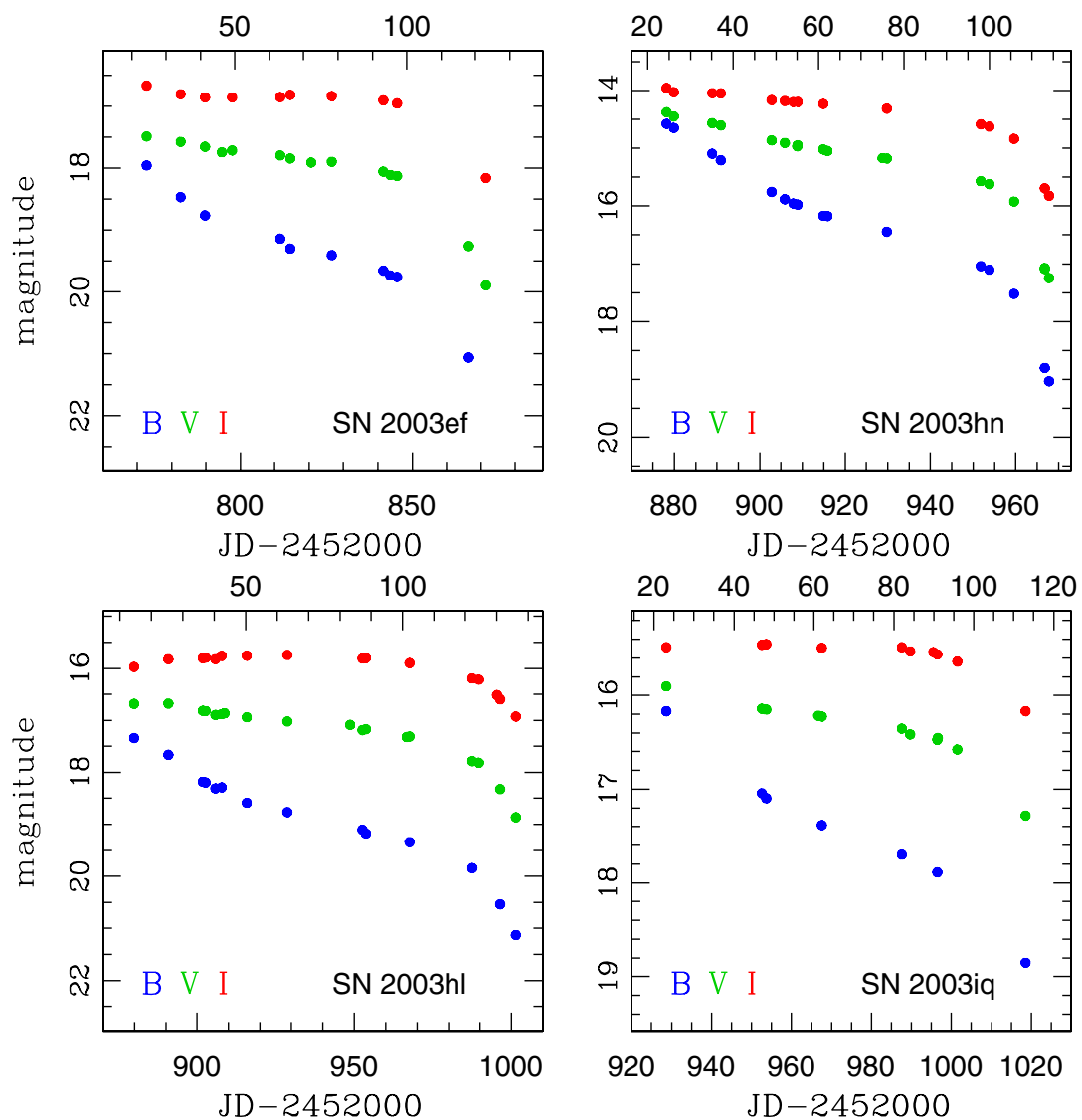

Figure 3. Optical light curves of four SNe during the first $\sim 120$ days of their evolution. The top axis of each panel gives the phase in days since the EPM explosion time, derived using the D05 models (see Table 5).

(A color version of this figure is available in the online journal.)

where $\theta_{i}$ and $v_{i}$ are the derived quantities measured at time $t_{i}$, which are estimated following the steps explained in the next sections. Equation (3) shows that the quantity $\theta / v$ increases linearly with time, so $D$ and $t_{0}$ can be derived from at least two spectroscopic and photometric observations. More observations allow us to check the internal consistency of the method.

\subsection{Dilution Factors}

The dilution factors correspond to the ratio of the luminosity of a SN atmosphere model $\left(L_{\lambda^{\prime}}\right)$ and the corresponding blackbody luminosity,

$$
\zeta_{\lambda^{\prime}}^{2}=\frac{L_{\lambda^{\prime}}}{\pi B_{\lambda^{\prime}}(T) 4 \pi R^{2}} .
$$

In practice, the dilution factors must be derived for the same filter subsets employed to determine the color temperature $(T)$ of a SN. In this work we focus on three different optical filter subsets $(\{B V\},\{B V I\}$, and $\{V I\})$, and we used two $\mathrm{SN}$ atmosphere models, those by Eastman et al. (1996, E96 hereafter) and Dessart \& Hillier (2005b, D05 hereafter), to compute the dilution factors. See also Dessart \& Hillier (2005a) for more details of the input parameters of the D05 models. Because the color temperature of each SN was determined from colors measured in the observer's rest frame, both the atmosphere models and the blackbody function must be redshifted; thus, the dilution factors must be computed for the specific redshift of each SN.

We computed $B, V, I$ synthetic magnitudes using 58 spectra from E96 atmosphere models and 138 spectra from D05
Table 1

Heliocentric and CMB Redshifts for the SNe Used in This Work

\begin{tabular}{lcccc}
\hline \hline SN & Host Galaxy & $\begin{array}{c}c z_{\text {helio }} \\
\left(\mathrm{km} \mathrm{s}^{-1}\right)\end{array}$ & Source & $\begin{array}{c}c z_{\mathrm{CMB}} \\
\left(\mathrm{km} \mathrm{s}^{-1}\right)\end{array}$ \\
\hline 1992ba & NGC 2082 & 1092 & here & 1245 \\
1999br & NGC 4900 & 960 & NED & 1285 \\
$1999 \mathrm{em}$ & NGC 1637 & 800 & L02 & 670 \\
$1999 \mathrm{gi}$ & NGC 3184 & 543 & here & 831 \\
$2002 \mathrm{gw}$ & NGC 0922 & 3117 & here & 2877 \\
$2003 \mathrm{~T}$ & UGC 04864 & 8368 & NED & 8662 \\
$2003 \mathrm{bl}$ & NGC 5374 & 4382 & NED & 4652 \\
$2003 \mathrm{bn}$ & 2MASX J10023529-2110531 & 3829 & NED & 4173 \\
$2003 \mathrm{ef}$ & NGC 4708 & 4440 & here & 4503 \\
$2003 \mathrm{hl}$ & NGC 0772 & 2265 & here & 2198 \\
$2003 \mathrm{hn}$ & NGC 1448 & 1347 & here & 1102 \\
$2003 \mathrm{iq}$ & NGC 0772 & 2364 & here & 2198 \\
\hline
\end{tabular}

Note.

${ }^{a}$ The NED values correspond to the redshifts of the host-galaxy nucleus, while the values measured in this work ("here") were measured from narrow emission lines of $\mathrm{H}$ II regions at the $\mathrm{SN}$ position. Also, L02 corresponds to the value adopted from Leonard et al. (2002b).

atmosphere models. For each filter subset $S$ (that is, $S=\{B V\}$, $\{B V I\},\{V I\}$ ), we fit blackbody functions in the $\mathrm{SN}$ rest frame $B_{\lambda^{\prime}}\left(T_{s}\right)$, and solved for $T_{s}$ and $\zeta_{S, z}$ by minimizing the quantity

$$
\epsilon=\sum_{\bar{\lambda} \in S}\left[M_{\bar{\lambda}}+5 \log \left(\frac{R}{10 \mathrm{pc}}\right)+5 \log \left(\zeta_{S, z}\right)-b_{\bar{\lambda}}\left(T_{S}, z\right)\right]^{2}
$$




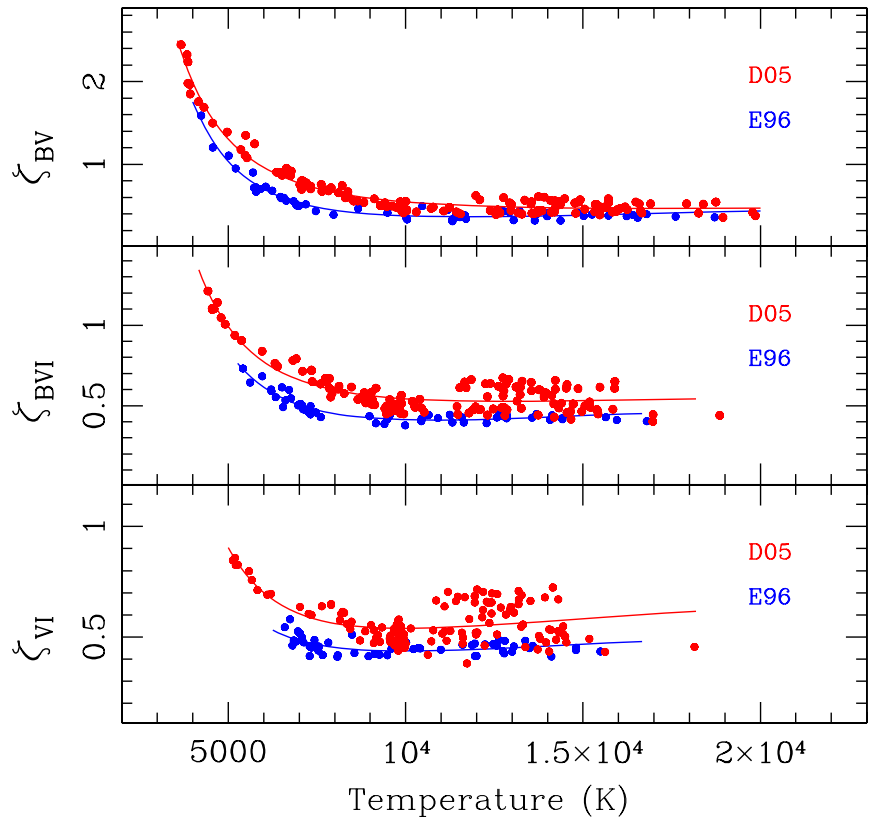

Figure 4. Dilution factors $\zeta$ as a function of the color temperature, computed at $z=0$ from the E96 (blue dots) and D05 (red dots) atmosphere models for three different filter subsets $(\{B V\},\{B V I\}$, and $\{V I\})$. The blue (red) line corresponds to the polynomial fit performed to the E96 (D05) dilution factors.

(A color version of this figure is available in the online journal.)

Table 2

Dilution Factor Coefficients and Dispersion

\begin{tabular}{lccccccccc}
\hline \hline Filter Subset & \multicolumn{4}{c}{ E96 } & & \multicolumn{5}{c}{ D05 } \\
\cline { 2 - 7 } \cline { 7 - 10 } & $b_{0}$ & $b_{1}$ & $b_{2}$ & $\sigma$ & & $b_{0}$ & $b_{1}$ & $b_{2}$ & $\sigma$ \\
\hline$\{B V\}$ & 0.756 & -0.900 & 0.520 & 0.048 & & 0.593 & -0.450 & 0.403 & 0.075 \\
$\{B V I\}$ & 0.733 & -0.693 & 0.373 & 0.027 & & 0.711 & -0.476 & 0.308 & 0.068 \\
$\{V I\}$ & 0.702 & -0.531 & 0.265 & 0.029 & 0.915 & -0.747 & 0.371 & 0.077 \\
\hline
\end{tabular}

Here $R$ is the photospheric radius, $M_{-}$is the redshifted synthetic absolute magnitude of the atmosphere model for a band with central wavelength $\bar{\lambda}$, and $b_{\bar{\lambda}}\left(T_{s}, z\right)$ is the synthetic magnitude of $\pi B_{\lambda^{\prime}}\left(T_{S}\right) 10^{-0.4\left[A(\lambda)+A^{\prime}\left(\lambda^{\prime}\right)\right]} /(1+z)$, given by

$$
b_{\bar{\lambda}}=-2.5 \log _{10} \int \frac{\pi \lambda B_{\lambda^{\prime}}\left(T_{s}\right) 10^{-0.4\left[A(\lambda)+A^{\prime}\left(\lambda^{\prime}\right)\right]}}{h c(1+z)} S(\lambda) d \lambda+Z P \text {, }
$$

where $S(\lambda)$ is the filter transmission function and $Z P$ is the zero point of the photometric system (Hamuy et al. 2001). The constants $h$ and $c$ are the Planck constant and the speed of light, respectively. Clearly, the dilution factors depend on the specific redshift of the $\mathrm{SN}$ and on the filter subset used to obtain temperature of the models. Figure 4 shows the resulting dilution factors versus temperature at $z=0$. We performed polynomial fits to $\zeta\left(T_{s}\right)$ of the form

$$
\zeta\left(T_{s}\right)=\sum_{j=0}^{2} b_{s, j}\left(\frac{10^{4} \mathrm{~K}}{T_{s}}\right)^{j}
$$

Table 2 lists the $b_{s, j}$ coefficients at $z=0$ for three filter subsets and both atmosphere models (E96 and D05). The corresponding polynomial fits are shown in Figure 4.

The D05 dilution factors are quite insensitive to the color temperature above $\sim 9000 \mathrm{~K}$, and lie around 0.5 , while at lower temperatures they increase sharply with decreasing temperature, reaching a value over unity below $\sim 5000 \mathrm{~K}$. The E96 dilution factors present the same pattern, but they are systematically lower than the D05 dilution factors by $\sim 15 \%$ The origin of these differences is unclear. Dessart \& Hillier (2005a) discuss that the discrepancy might be related to the different approach used to handle relativistic terms. Also, D05 solved the non-LTE (nonlocal thermodynamic equilibrium) problem for all the species, and employed a very complex atom model. E96, on the other hand, solved the non-LTE problem for a few species, while for the rest of the metals the excitation and ionization were assumed to be given by the Saha-Boltzmann equation, and the opacity was taken as pure scattering. Another important difference between the E96 and D05 dilution factors is the dependence on the parameters involved in the atmosphere modeling. While the E96 dilution factors show little sensitivity to a broad range of physical parameters other than temperature, the D05 models show a larger dispersion at a given color temperature. However, this is also due to D05 models covering a larger range of radii, density profiles $\left(\rho \propto r^{-n}\right)$ and temperature than E96. On average, the E96 models lead to a dispersion of $\sigma \sim 0.03$ in $\zeta$, while the D05 models yield $\sigma \sim 0.07$.

\subsection{Angular Radii}

An apparent angular radius $\left(\theta \zeta_{s}\right)$ and a color temperature $\left(T_{s}\right)$ of the SN can be obtained by fitting a Planck function $B_{\lambda^{\prime}}\left(T_{S}\right)$ to the observed broadband magnitudes (see Equation 1). Here $S$ is the filter subset combination, i.e., $S=\{B V\},\{B V I\},\{V I\}$. Since we have two unknowns $\left(\theta \zeta_{s}, T_{s}\right)$, the subsets must contain at least two filters. In order to derive these parameters, we used a least-squares technique at each spectroscopic observation epoch (see Section 3.6) by minimizing the quantity

$$
\chi^{2}=\sum_{s} \frac{\left[m_{\bar{\lambda}}+5 \log \left(\theta \zeta_{s, z}\right)-b_{\bar{\lambda}}\left(T_{s}, z\right)\right]^{2}}{\sigma_{m}^{2}}
$$

Here, $m_{\bar{\lambda}}$ is the apparent magnitude in the filter with central wavelength $\bar{\lambda}$ (i.e., $\left.m_{\bar{\lambda}} \in\{B, V, I\}\right), \sigma_{m}$ is the photometric error in the magnitude $m_{\bar{\lambda}}$, and $b_{\bar{\lambda}}$ is defined in Equation 6. Because $\zeta_{s}$ is mainly a function of the color temperature (Figure 4), it is possible to use $T_{s}$ to solve for $\zeta_{s}$ and determine the true angular radius $\theta$ from $\theta \zeta_{s}$.

\subsection{Physical Radii}

Once $\theta$ is determined, the next step is to measure the photospheric velocity (see Equation 3 ). The photospheric velocity of the $\mathrm{SN}$ at a given epoch can be obtained from the absorption lines in the spectra. We measured velocities ${ }^{9}$ from the minima of $\mathrm{H} \alpha, \mathrm{H} \beta, \mathrm{H} \gamma$, and $\mathrm{Fe}$ II $\lambda 5169$ lines, for all $12 \mathrm{SNe}$. See Jones (2008) for tables that list the spectroscopic velocities. Figures 5-7 show the temporal evolution of the spectral line velocities.

To date the photospheric velocities have been estimated using weak spectral absorption features such as Fe II $\lambda \lambda 5169,5018$, 4924, and Sc II $\lambda 4670$ (Schmidt et al. 1992; Leonard et al. $2002 \mathrm{~b}$ ). The physical assumption is that these lines are weak

\footnotetext{
9 We employed the nonrelativistic formulae to derive the expansion velocities from the Doppler shift of the absorption lines. This approach is reasonable because the highest velocity used in this work is $\sim 0.035 c$, for which the difference in the velocity using the relativistic and nonrelativistic formulae is less than $2 \%$. However, typical velocities employed in the EPM analysis are of $0.02 c$, for which the difference is $\sim 1 \%$.
} 

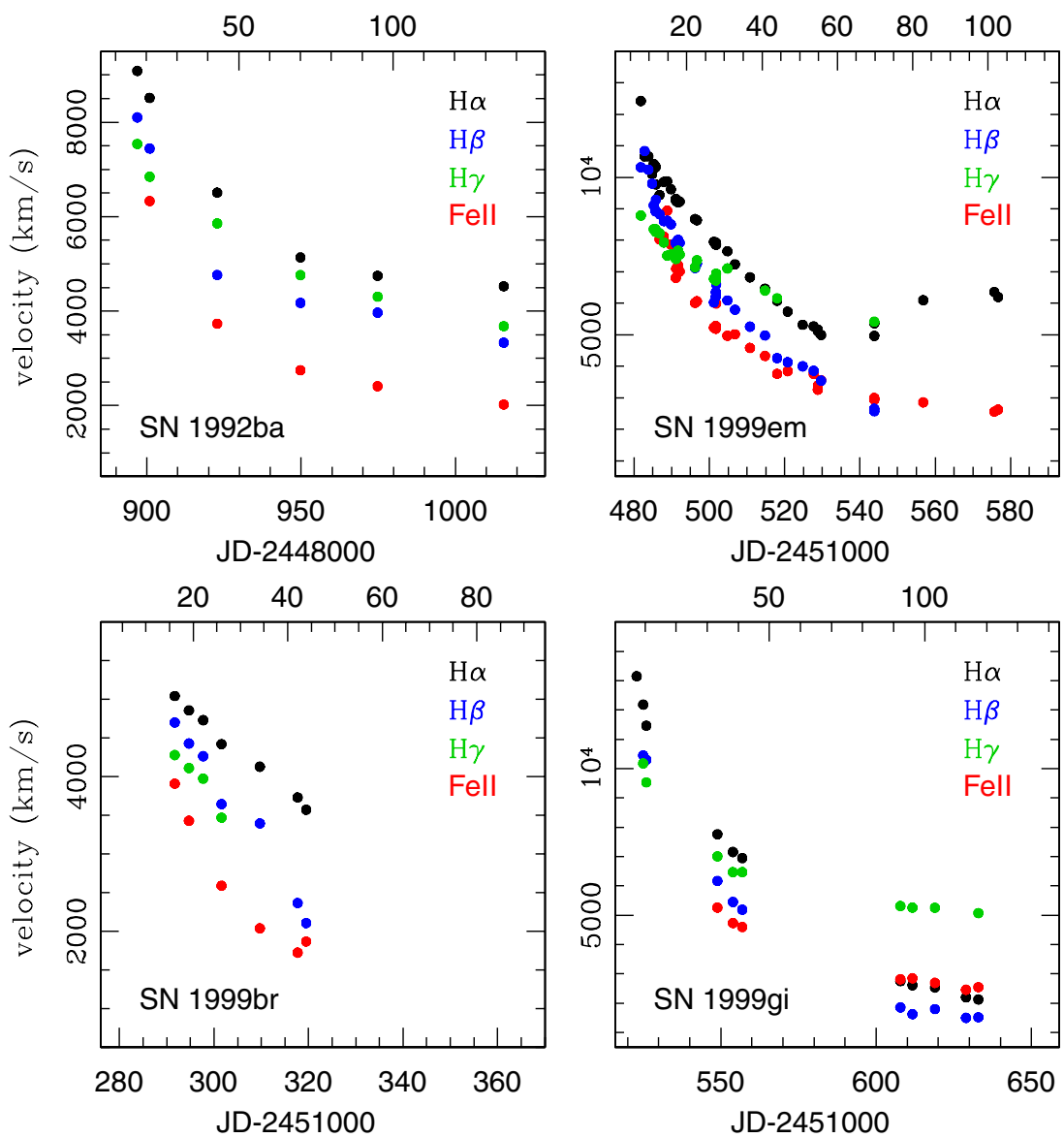

Figure 5. Line velocity evolution determined from the P Cygni absorption minima of four different features during $\sim 100$ days after discovery. The top axis of each panel gives the phase in days since the EPM explosion time derived using the D05 models (see Table 5).

(A color version of this figure is available in the online journal.)

and formed near the photosphere of the SN. However, there are two problems with this approach: (1) at early times the spectra are dominated by Balmer lines and the weak lines are absent, and (2) the synthetic spectra show that even the weak lines do not necessarily yield true photospheric velocities (Dessart \& Hillier 2006). One way to circumvent these problems is to use the Balmer lines which are present in the spectra over most of the evolution of the SN. Although the Balmer lines are optically thicker than the Fe II lines, Dessart \& Hillier (2006) argue that, contrary to what is usually believed, optically thick lines do not necessarily overestimate the photospheric velocity, and the offset from the photospheric velocity can be measured from the synthetic spectra. In this work we decided to use the minimum of the $\mathrm{H} \beta$ absorption line to derive the photospheric velocity because this line is present during the entire plateau phase, it can be easily identified, and it does not present any blend, at least in the first $\sim 50$ days after explosion.

To convert from observed $\mathrm{H} \beta$ spectroscopic velocities to true photospheric velocities we used the synthetic spectra from E96 and D05. Figure 8 shows (in red) the ratio of $\mathrm{H} \beta$ velocity and the photospheric velocity, as a function of $\mathrm{H} \beta$ velocity for all of the D05 models. Note that the D05 models predict that the $\mathrm{H} \beta$ line forms quite close to the photosphere at all epochs (for all values of $v_{H \beta}$ ). Also plotted in Figure 8 (in blue) are the E96 models which confirm that the $\mathrm{H} \beta$ forms close to the photosphere at early epochs, when $v_{H \beta}$ is high. However, at later epochs (lower $v_{H \beta}$ ) E96 predict that $\mathrm{H} \beta$ forms in outer layers (higher velocities) than D05. It is also important to note that the E96 models cover a shorter range in velocity $(\sim 4500$ $\left.12,000 \mathrm{~km} \mathrm{~s}^{-1}\right)$ than the D05 models $\left(\sim 2000-17,000 \mathrm{~km} \mathrm{~s}^{-1}\right)$, which restricts the EPM analysis using the E96 models.

To derive the ratio between the $\mathrm{H} \beta$ and the photospheric velocity we used a polynomial fit of the form

$$
\frac{v_{H \beta}}{v_{\text {phot }}}=\sum_{j=0}^{2} a_{j}\left(v_{H \beta}\right)^{j},
$$

(see Figure 8). The $a_{j}$ coefficients are listed in Table 3. The E96 models lead to a dispersion of $\sigma=0.06$ and the D05 models to $\sigma=0.04$. The photospheric velocity $v_{i}$ can be obtained from a measurement of $v_{H \beta}$ :

$$
v_{i}=\frac{v_{H \beta}}{\sum_{j=0}^{2} a_{j}\left(v_{H \beta}\right)^{j}} .
$$

In order to examine which of the adopted photospheric velocity conversions was closer to reality, we compared the ratio between the $\mathrm{H} \alpha$ and $\mathrm{H} \beta$ velocities measured from the observed spectra of our sample of $\mathrm{SNe}$ and from the synthetic spectra of the E96 and D05 models. Figure 9 shows the $\mathrm{H} \alpha / \mathrm{H} \beta$ velocity ratio as a function of the $\mathrm{H} \beta$ velocity. It can be seen that, while there is good agreement between theory and 

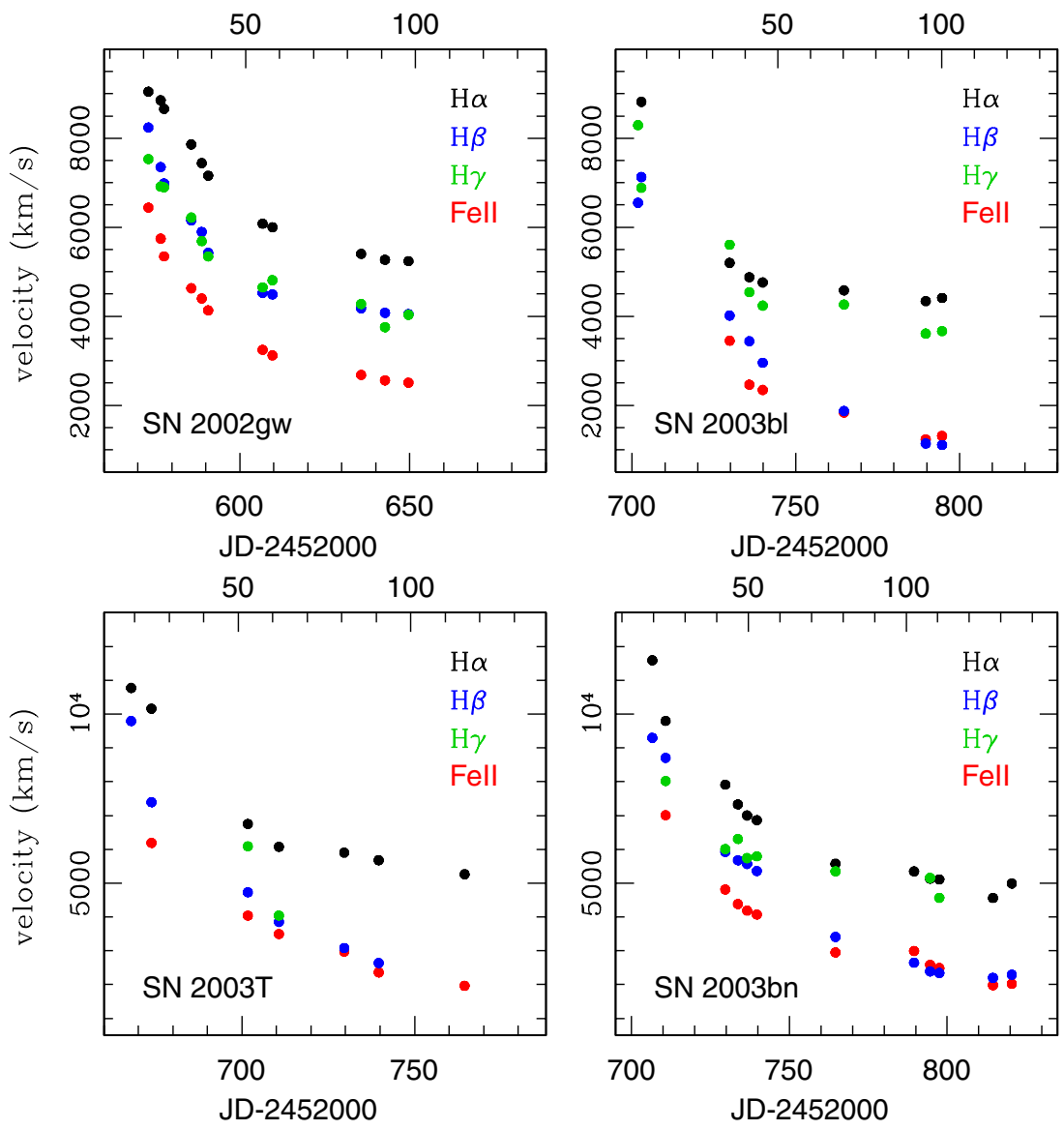

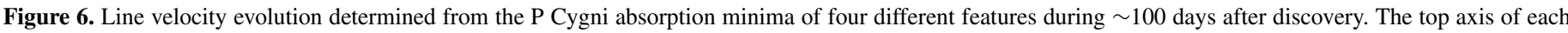
panel gives the phase in days since the EPM explosion time derived using the D05 models (see Table 5).

(A color version of this figure is available in the online journal.)

observations at high $\mathrm{H} \beta$ velocities $\left(\sim 7000-10,500 \mathrm{~km} \mathrm{~s}^{-1}\right)$, the D05 models underestimate the $\mathrm{H} \alpha$ velocities (or overestimate the $\mathrm{H} \beta$ velocities) at lower expansion velocities. This could be due to time-dependence effects that are not included in the D05 models that assume steady state (Dessart \& Hillier 2008). On the other hand, the $\mathrm{H} \alpha / \mathrm{H} \beta$ velocity ratio predicted by the E96 models is in good agreement with the observations at all $\mathrm{H} \beta$ velocities, although there are few models below $\sim 6000$ $\mathrm{km} \mathrm{s}^{-1}$ to draw strong conclusions. This suggests that E96 predict more realistic line profiles in the SN ejecta than D05 and therefore should provide a better photospheric velocity conversion.

\subsection{Extinction}

To estimate the amount of Galactic foreground extinction we used the IR dust maps of Schlegel et al. (1998). Table 4 summarizes the foreground extinction adopted. In this work we used two different methods for the determination of host-galaxy reddenings of our SN sample: a spectroscopic method (DES hereafter), and a method based on the color evolution of the $\mathrm{SNe}$ (OLI hereafter). The former consists in fitting different model spectra to the early-time spectra of a SN. The two fitting parameters are the amount of reddening and the photospheric temperature (Dessart \& Hillier 2006; Dessart et al. 2008). The color-based technique was developed by Olivares (2008) and is based on the assumption that the color at the end of the plateau phase is the same for all SNe IIP. In both cases we adopted the Cardelli et al. (1989) extinction law (with $R_{V}=3.1$ ).
Table 4 lists the host-galaxy visual extinction values $A_{V}$ obtained from both methods. Also, Figure 10 shows the OLI versus DES visual extinctions. As can be seen, there are no systematic differences between the models. However, there are individual differences, especially in five $\mathrm{SNe}$, whose names are explicitly marked in the plot.

\subsection{Implementation of EPM}

The EPM method is only valid in the optically thick phase of a H-rich expanding atmosphere. Observationally, this period corresponds to the plateau phase of Type II SNe and thus justifies our first selection criterion in Section 2.3.

The EPM requires at least two simultaneous photometric and spectroscopic observations (see Equation 3), but we recommend the use of at least three points in order to obtain an internal check. The photometry is used to determine the angular size of the SN and the spectroscopy is used to measure the expansion velocities of the SN. The requirement of simultaneous photometric and spectroscopic observations is usually not accomplished; the photometry and the spectroscopy of a SN are taken at different epochs. To overcome this problem, it is necessary to interpolate the photometry or the velocities measured from the spectra. In this work we decided to interpolate the photometry for two reasons: (1) the number of photometric observations in our sample of $\mathrm{SNe}$ is far greater than the number of spectroscopic observations, and (2) the optical apparent magnitude of SNe II-P is nearly constant during the plateau phase, making the photometry interpolation more reliable than the velocity interpolation, 

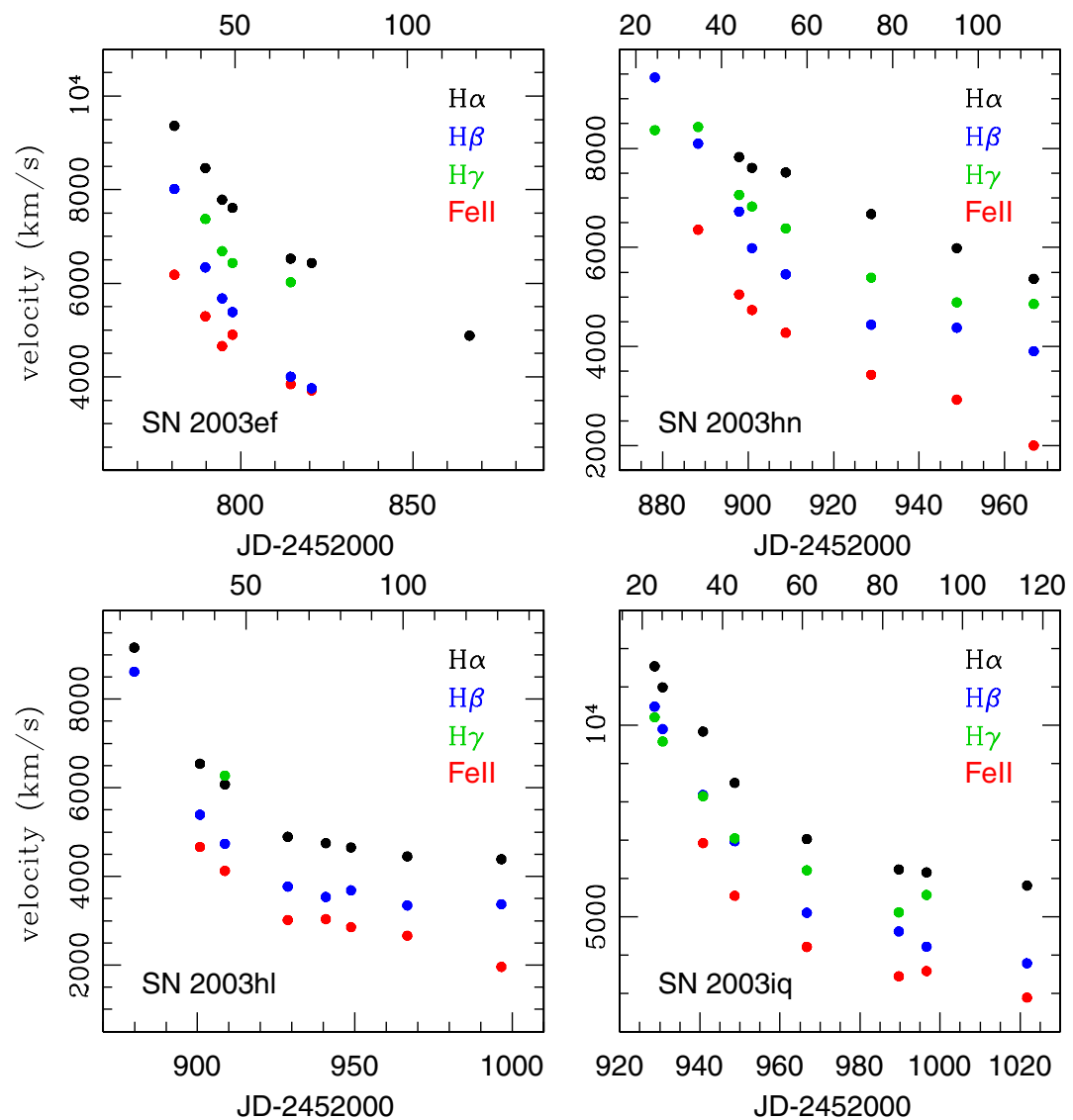

Figure 7. Line velocity evolution determined from the P Cygni absorption minima of four different features during $\sim 100$ days after discovery. The top axis of each panel gives the phase in days since the EPM explosion time derived using the D05 models (see Table 5).

(A color version of this figure is available in the online journal.)

which has a steeper dependence with time. To interpolate a magnitude at the epoch of a given spectroscopic observation, we use a quadratic polynomial fit, using four photometric observations around the spectroscopic date.

In this study, we restricted the EPM analysis to the first $\sim 45$ 50 days after explosion because there are clear departures from linearity in the $\theta / v$ versus $t$ plots after this date. Dessart \& Hillier (2005b) show that the D05 models are poor at late time and therefore should not be used for such epochs, supporting this restriction. In Figure 11 we plot the EPM solutions for SN 1999em (because it has extensive photometric and spectroscopic observations during the plateau phase) using the $\{B V\},\{B V I\}$, and $\{V I\}$ filter subsets and the D05 models. The solid line corresponds to the least-squares fit to the derived EPM quantities using the first $\sim 70$ days after explosion, while the dashed line corresponds to the least-squares fit using only the first $\sim 40$ days after explosion. As can be noted, after $\sim 40$ days from explosion (marked with a red triangle) there is departure from the linear $\theta / v$ versus $t$ relation in all three cases. This justifies our second and third selection criteria in Section 2.3. However, this restriction severely lowers the number of SNe of our sample to which we can apply the EPM. Out of the initial $51 \mathrm{SNe}$ of the M. Hamuy (2009, in preparation) sample, only 11 objects fulfill the requirement of having a plateau behavior and having early-time photometry and spectroscopy for the EPM analysis.

\subsubsection{EPM Analysis of Individual SNe}

We present here the EPM analysis of 12 SNe IIP (11 from our database and one from the literature) with early spectroscopic and photometric observations. We carried out the analysis using three different filter subsets $(\{B V\},\{B V I\}$, and $\{V I\})$, two sets of host-galaxy extinction (OLI, DES), and two atmosphere models (E96, D05), yielding a total of 12 solutions for each $\mathrm{SN}$. The tables that summarize the EPM quantities are available in electronic format for all 144 cases. In the remainder of this section we restrict the presentation to the 6 solutions that use the DES extinction because they give the lowest dispersion in the Hubble diagrams. Figures 12-23 show these six solutions for each of the $12 \mathrm{SNe}$. Below, we provide the EPM distance $D$ and the explosion date $t_{0}$ and their uncertainties, using DES and the VI filter subset, and we compare the time of explosion to the range restricted by pre-SN images of the host galaxies. These results are also summarized in Table 5.

In order to obtain a more realistic estimation of the uncertainty in the distance and the explosion date, we computed 100 Monte Carlo simulations for each SN, in which we varied all the parameters involved in the EPM (see Table 6), and we averaged the 100 distances and explosion dates to derive the EPM values of $D$ and $t_{0}$. This produces small differences between the results computed from the initial single EPM solution and that obtained from the 100 Monte Carlo simulations, but the latter provides a much more realistic estimate of the uncertainties.

$S N$ 1992ba. Figure 12 shows $\theta / v$ versus time for SN 1992ba using the $\{B V\},\{B V I\}$, and $\{V I\}$ filter subsets and the E96 and D05. We used three epochs (JD 2448896.9-2448922.8) to compute the distance to this SN. In order to use the velocities measured on JD 2448896.9 and 2448900.9 , we had to extrapolate the $I$-band photometry until JD 2448896.9 . 


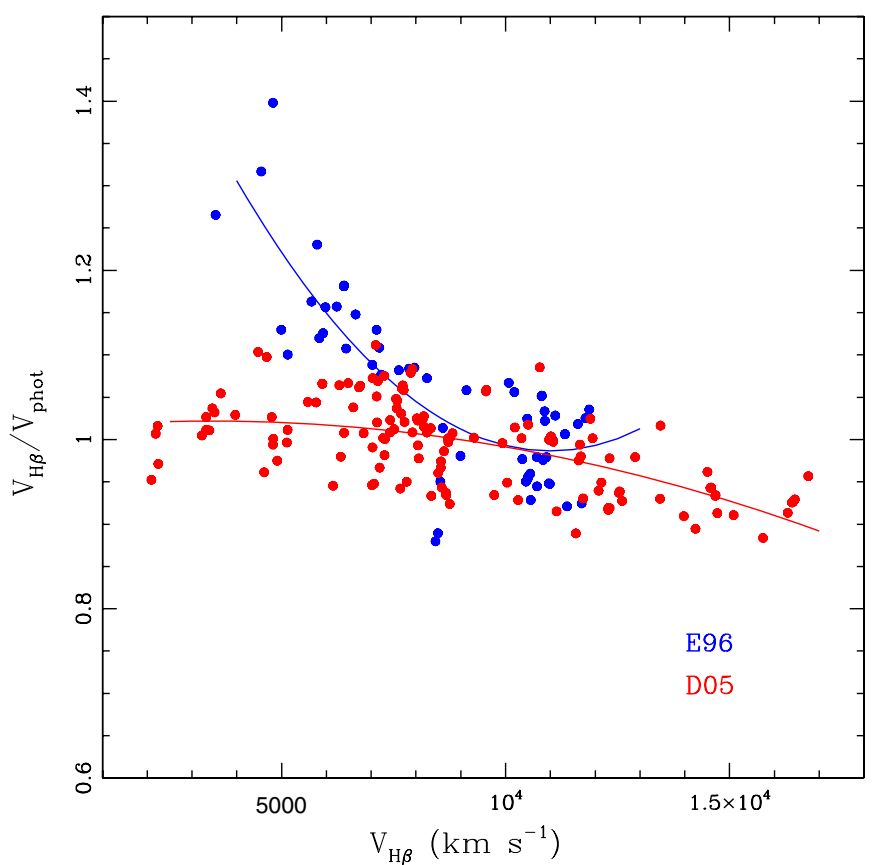

Figure 8. Ratio of the $\mathrm{H} \beta$ velocity to the photospheric velocity versus the $\mathrm{H} \beta$ velocity of the individual SN models. The blue dots correspond to E96 models and the red dots to D05 models. The blue (red) line corresponds to the polynomial fit performed to the E96 (D05) photospheric velocity conversion. (A color version of this figure is available in the online journal.)

Table 3

$\mathrm{H} \beta$ to Photospheric Velocity Ratio Coefficients and Dispersion

\begin{tabular}{lcc}
\hline \hline$j$ & $a_{j}(\mathrm{E} 96)$ & $a_{j}(\mathrm{D} 05)$ \\
\hline 0 & 1.775 & 1.014 \\
1 & $-1.435 \times 10^{-4}$ & $4.764 \times 10^{-6}$ \\
2 & $6.523 \times 10^{-9}$ & $-7.015 \times 10^{-10}$ \\
$\sigma$ & 0.06 & 0.04 \\
\hline
\end{tabular}

Table 4

SN Host Galaxy and Galactic Extinction Adopted

\begin{tabular}{lccc}
\hline \hline SN & $\begin{array}{c}A_{V}(\mathrm{OLI})^{\mathrm{a}} \\
\text { Host }\end{array}$ & $\begin{array}{c}A_{V}(\mathrm{DES})^{\mathrm{b}} \\
\text { Host }\end{array}$ & $\begin{array}{c}A_{V}(\mathrm{IR} \text { maps })^{\mathrm{c}} \\
\text { Galactic }\end{array}$ \\
\hline 1992ba & $0.30(0.15)$ & $0.43(0.16)$ & $0.193(0.031)$ \\
$1999 \mathrm{br}$ & $0.94(0.20)$ & $0.25(0.16)$ & $0.078(0.012)$ \\
$1999 \mathrm{em}$ & $0.24(0.14)$ & $0.31(0.16)$ & $0.134(0.021)$ \\
$1999 \mathrm{gi}$ & $1.02(0.15)$ & $0.56(0.16)$ & $0.055(0.009)$ \\
$2002 \mathrm{gw}$ & $0.18(0.16)$ & $0.40(0.19)$ & $0.065(0.010)$ \\
$2003 \mathrm{~T}$ & $0.35(0.15)$ & $0.53(0.31)$ & $0.104(0.017)$ \\
$2003 \mathrm{bl}$ & $0.26(0.15)$ & $0.00(0.16)$ & $0.090(0.014)$ \\
$2003 \mathrm{bn}$ & $-0.04(0.15)$ & $0.09(0.16)$ & $0.215(0.034)$ \\
$2003 \mathrm{ef}$ & $0.98(0.15)$ & $1.24(0.25)$ & $0.153(0.024)$ \\
$2003 \mathrm{hl}$ & $1.72(0.18)$ & $1.24(0.25)$ & $0.241(0.039)$ \\
$2003 \mathrm{hn}$ & $0.46(0.14)$ & $0.59(0.25)$ & $0.047(0.008)$ \\
$2003 \mathrm{iq}$ & $0.25(0.16)$ & $0.37(0.16)$ & $0.241(0.039)$ \\
\hline
\end{tabular}

Notes.

a Olivares (2008).

b Dessart \& Hillier (2006) and Dessart et al. (2008).

${ }^{c}$ Schlegel et al. (1998).

SN 1992ba was discovered by Evans (1992) on JD 2448896.3. McNaught (1992) reported that the SN was not present on a plate taken on JD 2448883.2 with limiting magnitude 19. The EPM solution yields $t_{0}=\mathrm{JD} 2448883.9 \pm 3.0$ using the E96 models and $t_{0}=\mathrm{JD} 2448879.8 \pm 5.6$ with D05. These results agree

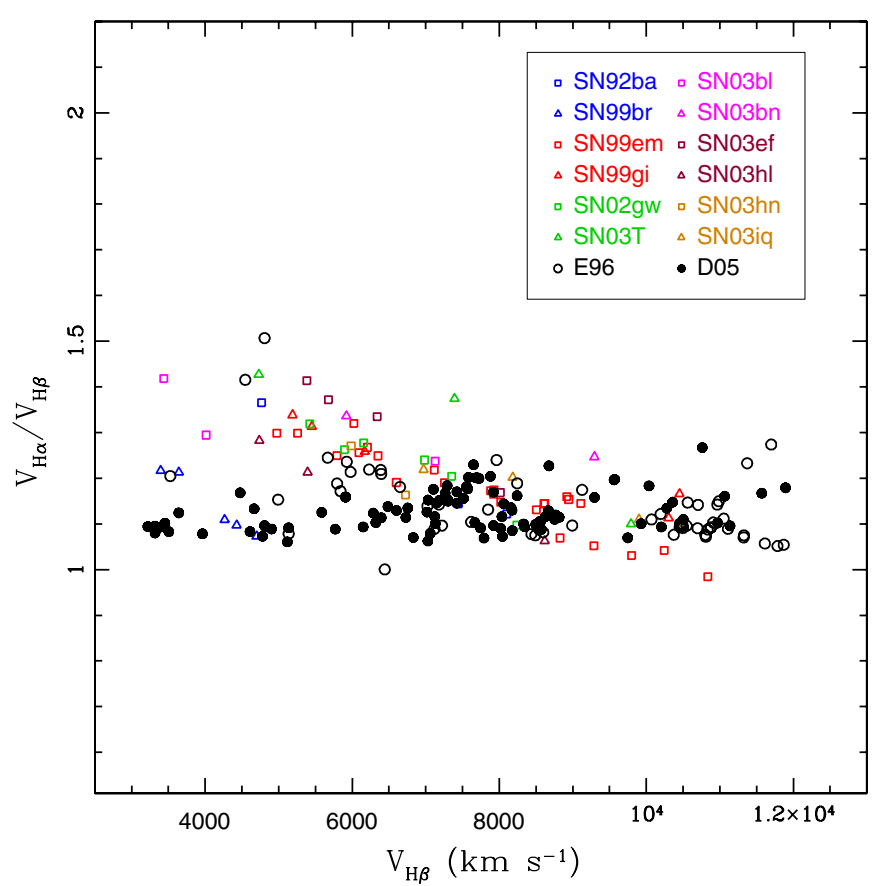

Figure 9. Ratio of the $\mathrm{H} \alpha$ velocity to the $\mathrm{H} \beta$ velocity as a function of the $\mathrm{H} \beta$ velocity. The triangles and the squares represent velocities measured from the spectra of our SN sample. The open and filled black circles correspond to the velocity ratio measured from the synthetic spectra of E96 and D05, respectively. (A color version of this figure is available in the online journal.)

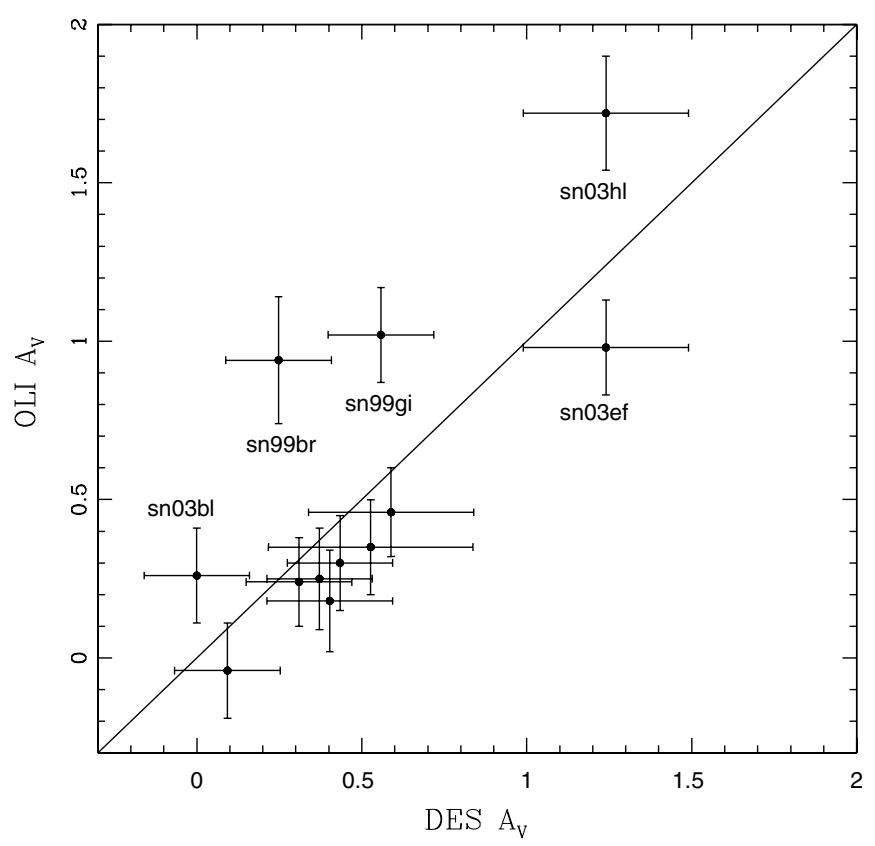

Figure 10. Comparison between the DES and OLI reddening methods for the $12 \mathrm{SNe}$. The straight line has a slope of unity. The more deviant SNe are labeled.

(within $1 \sigma$ ) with the explosion date constrained by the preand post-explosion observations. The distances derived to SN 1992ba are $D=16.4 \pm 2.5 \mathrm{Mpc}$ and $D=27.2 \pm 6.5 \mathrm{Mpc}$ using the E96 and the D05 dilution factors, respectively.

$S N$ 1999br. Figure 13 shows $\theta / v$ versus time for $\mathrm{SN}$ $1999 \mathrm{br}$ using the $\{B V\},\{B V I\}$, and $\{V I\}$ filter subsets and the D05 atmosphere models. We used five epochs (JD 2451291.7-2451309.7) to compute the distance to this SN. The EPM solution shows some departure from linearity using the $B V$ 


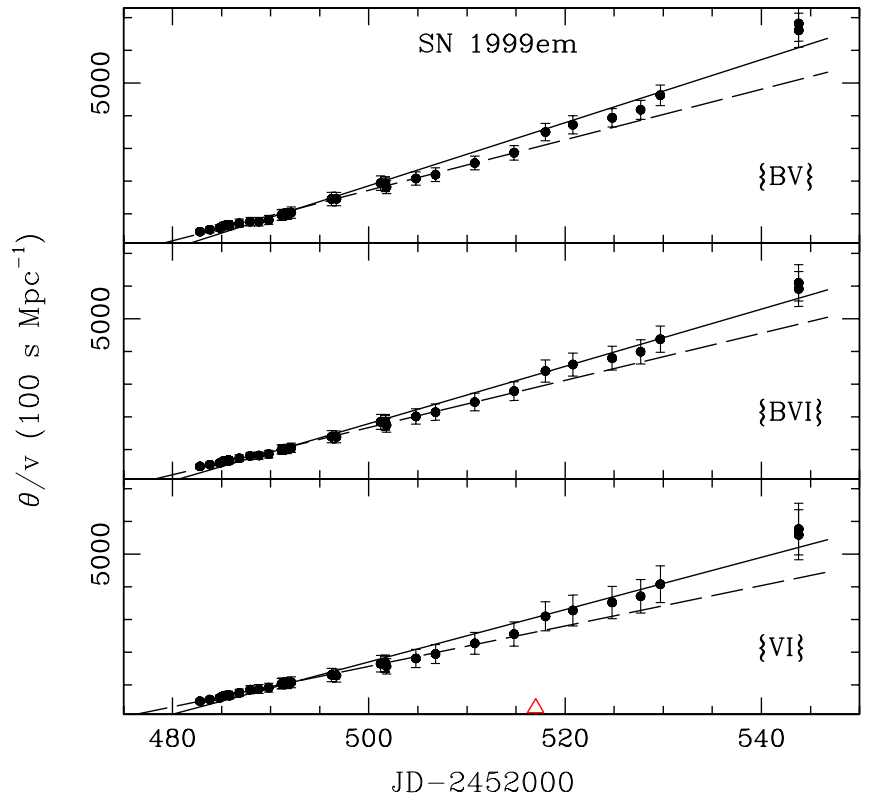

Figure 11. The ratio $\theta / v$ as a function of time for $\mathrm{SN} 1999 \mathrm{em}$ using the $\{B V\}$, $\{B V I\}$, and $\{V I\}$ filter subsets and the D05 models. The solid (dashed) lines correspond to unweighted least-squares fits to the derived EPM quantities using $\sim 70$ (40) days after explosion. The red triangle in the bottom panel shows day $\sim 40$ after explosion.

(A color version of this figure is available in the online journal.)

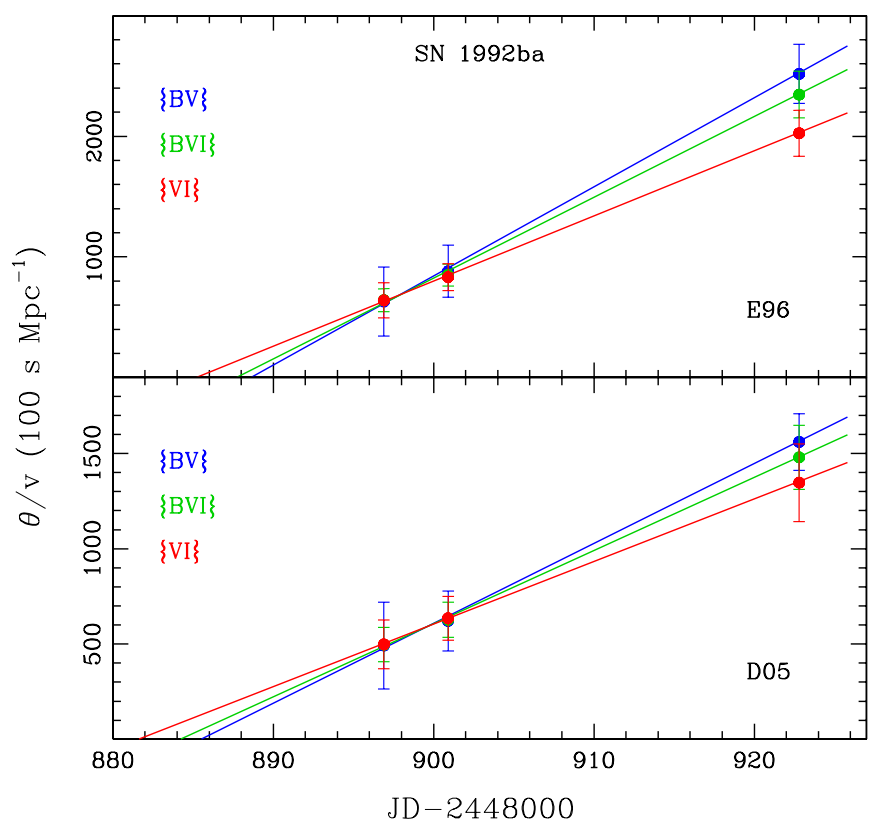

Figure 12. The ratio $\theta / v$ as a function of time for SN 1992ba using the $\{B V\}$, $\{B V I\}$, and $\{V I\}$ filter subsets. The ridge lines correspond to unweighted leastsquares fits to the derived EPM quantities. The upper and lower panels show the results using E96 and D05 dilution factors, respectively. In all cases we employ the DES reddening.

(A color version of this figure is available in the online journal.)

and $B V I$ filter subsets. SN 1999br exhibits very low expansion velocities, so we were unable to obtain its distance using the E96 models. This is because the photospheric velocity conversion factor $V_{H \beta} / V_{\text {phot }}$ is not defined at low expansion velocities (see Section 3.4 and Figure 8). The EPM solution yields $t_{0}=$ JD $2451275.6 \pm 7.7$ using the D05 models. This result compares very well with the observations, because SN 1999br was

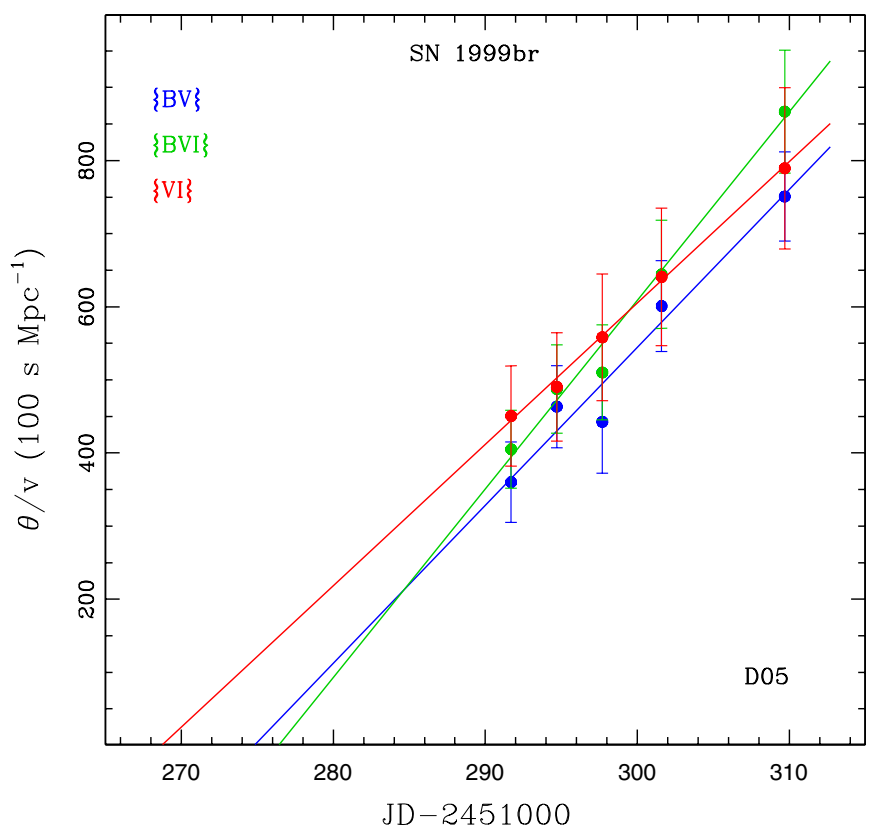

Figure 13. The ratio $\theta / v$ as a function of time for SN 1999br using the $\{B V\}$, $\{B V I\}$, and $\{V I\}$ filter subsets and the D05 models. The ridge lines correspond to unweighted least-squares fits to the derived EPM quantities. In all cases we employ the DES reddening.

(A color version of this figure is available in the online journal.)

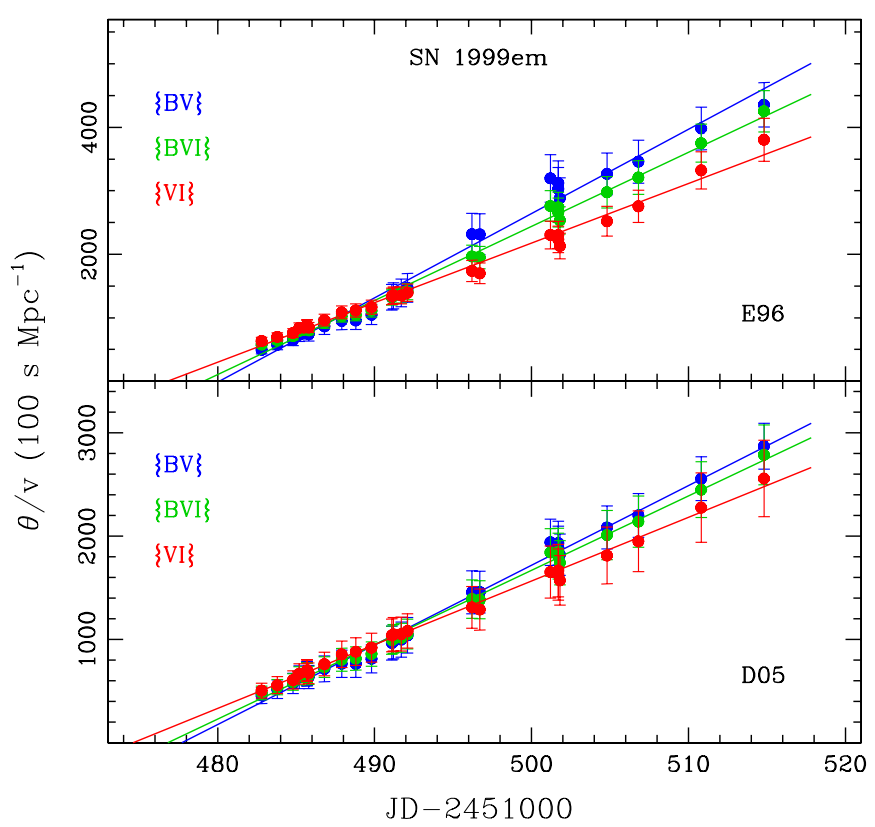

Figure 14. The ratio $\theta / v$ as a function of time for SN $1999 \mathrm{em}$ using the $\{B V\}$, $\{B V I\}$, and $\{V I\}$ filter subsets. The ridge lines correspond to unweighted leastsquares fits to the derived EPM quantities. The upper and lower panels show the results using E96 and D05 dilution factors, respectively. In all cases we employ the DES reddening.

(A color version of this figure is available in the online journal.)

discovered by the Lick Observatory Supernova Search (LOSS; Filippenko et al. 2001) with the Katzman Automatic Imaging Telescope (KAIT) on JD 2451280.9 (King 1999). An image taken on JD 2451264.9 showed nothing at the SN position at a limiting magnitudes of 18.5 ( $\mathrm{Li}$ 1999a). The EPM distance to SN 1999br is $D=39.5 \pm 13.5 \mathrm{Mpc}$ using the D05 dilution factors. 


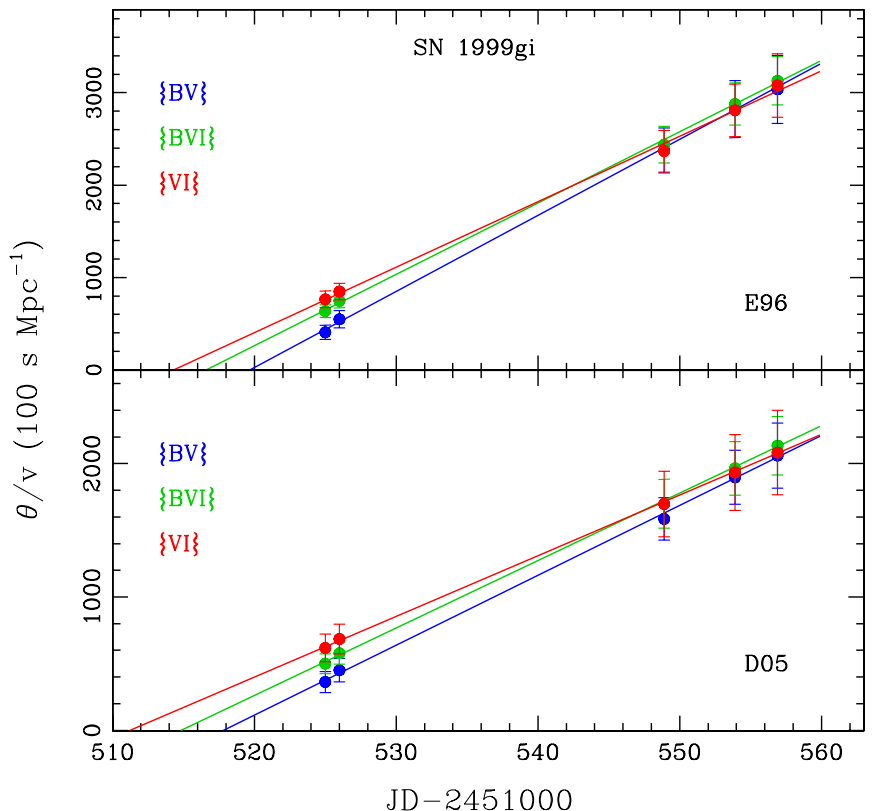

Figure 15. The ratio $\theta / v$ as a function of time for SN 1999gi using the $\{B V\}$, $\{B V I\}$, and $\{V I\}$ filter subsets. The ridge lines correspond to unweighted leastsquares fits to the derived EPM quantities. The upper and lower panels show the results using E96 and D05 dilution factors, respectively. In all cases we employ the DES reddening.

(A color version of this figure is available in the online journal.)

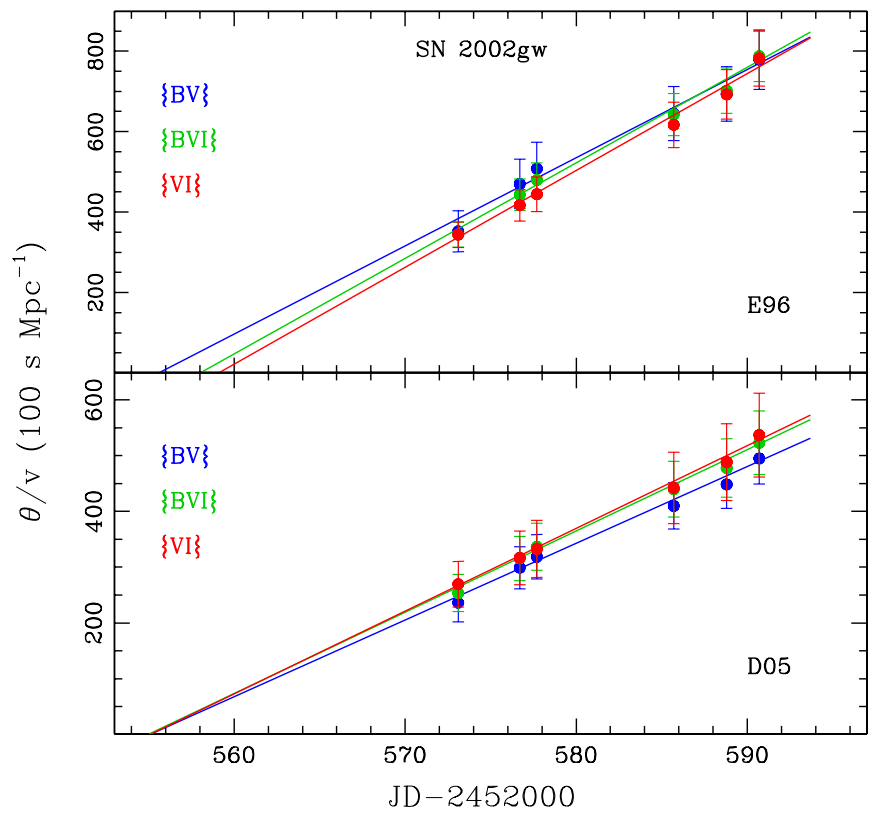

Figure 16. The ratio $\theta / v$ as a function of time for $\mathrm{SN} 2002 \mathrm{gw}$ using the $\{B V\}$, $\{B V I\}$, and $\{V I\}$ filter subsets. The ridge lines correspond to unweighted leastsquares fits to the derived EPM quantities. The upper and lower panels show the results using E96 and D05 dilution factors, respectively. In all cases we employ the DES reddening.

(A color version of this figure is available in the online journal.)

SN 1999em. SN 1999em is the best-ever observed SN IIP. Many photometric and spectroscopic observations were made by different observers during the plateau phase. Figure 14 shows $\theta / v$ versus time for the $\mathrm{SN} 1999 \mathrm{em}$ using the $\{B V\},\{B V I\}$, and $\{V I\}$ filter subsets and the E96 and D05 models. Table 7 summarizes the EPM quantities derived from the $\{V I\}$ filter

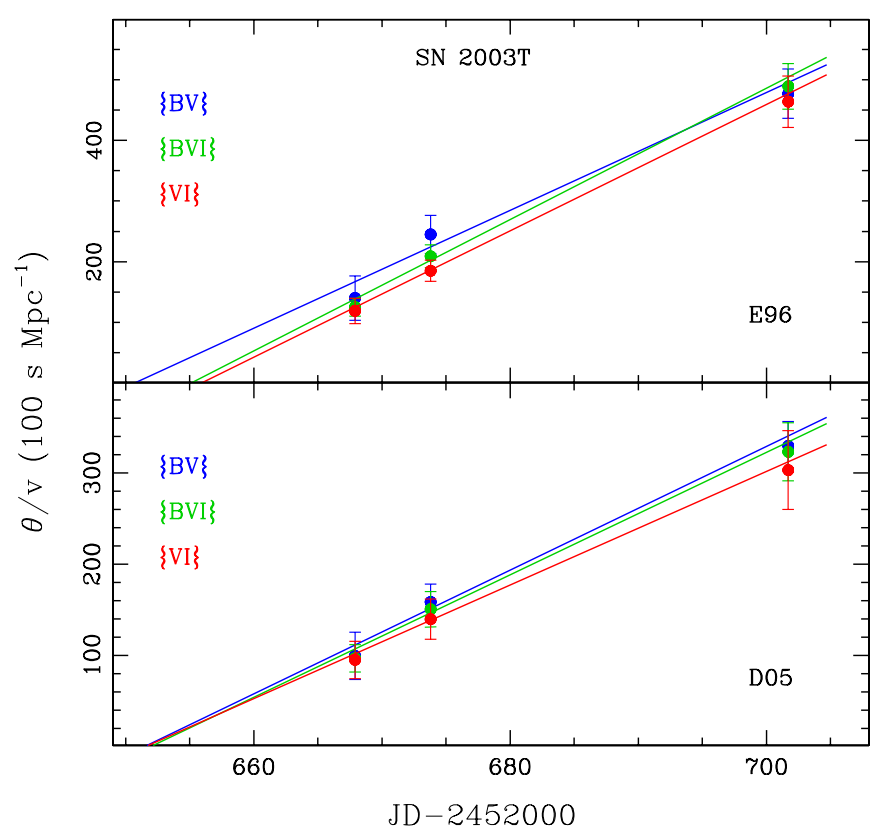

Figure 17. The ratio $\theta / v$ as a function of time for SN 2003T using the $\{B V\}$, $\{B V I\}$, and $\{V I\}$ filter subsets. The ridge lines correspond to unweighted leastsquares fits to the derived EPM quantities. The upper and lower panels show the results using E96 and D05 dilution factors, respectively. In all cases we employ the DES reddening.

(A color version of this figure is available in the online journal.)

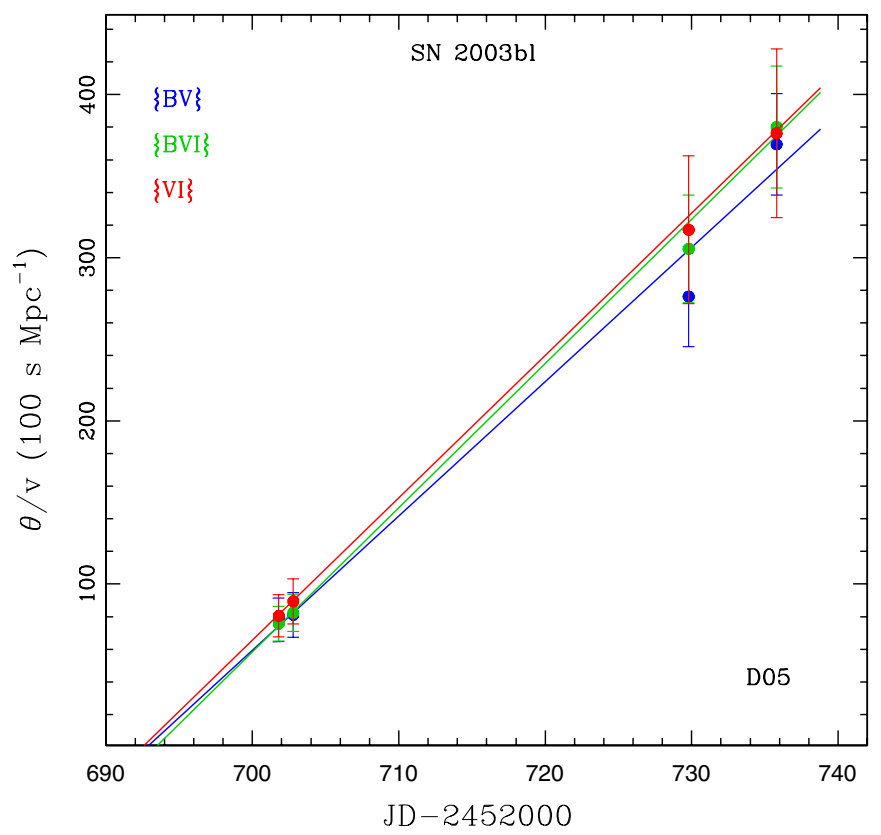

Figure 18. The ratio $\theta / v$ as a function of time for SN 2003bl using the $\{B V\}$, $\{B V I\}$, and $\{V I\}$ filter subsets and the D05 models. The ridge lines correspond to unweighted least-squares fits to the derived EPM quantities. In all cases we employ the DES reddening.

(A color version of this figure is available in the online journal.)

subset. We used 25 epochs (JD 2451482.8-2451514.8) to derive the distance to SN 1999em. Four spectra were taken from Hamuy et al. (2001) and the other 21 from Leonard et al. (2002b). In some cases there were two spectra taken at the same epoch from both sources; we used them individually in the EPM solution instead of averaging the measured velocities from each spectrum. We removed the first spectrum (JD 2451481.8) 


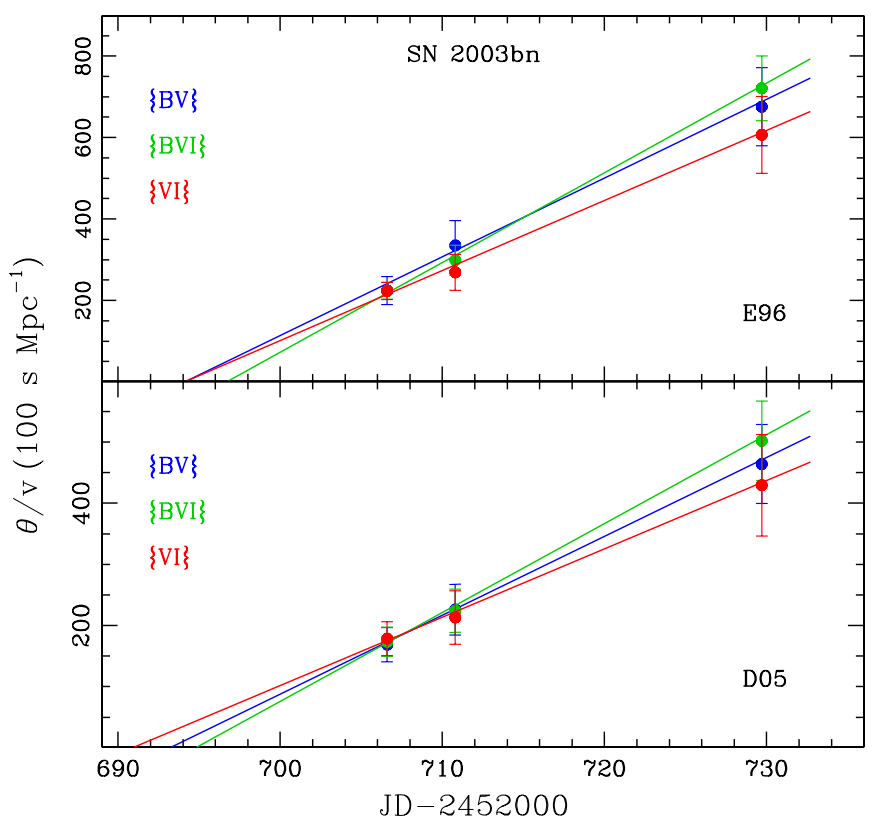

Figure 19. The ratio $\theta / v$ as a function of time for SN 2003bn using the $\{B V\}$, $\{B V I\}$, and $\{V I\}$ filter subsets. The ridge lines correspond to unweighted leastsquares fits to the derived EPM quantities. The upper and lower panels show the results using E96 and D05 dilution factors, respectively. In all cases we employ the DES reddening.

(A color version of this figure is available in the online journal.)

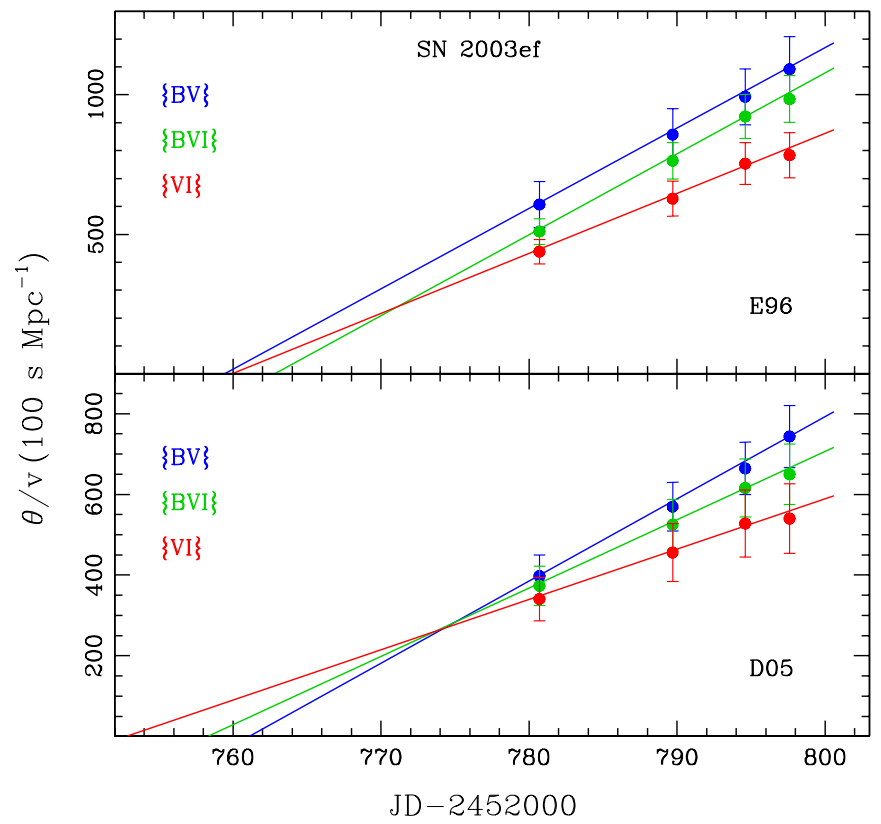

Figure 20. The ratio $\theta / v$ as a function of time for SN 2003ef using the $\{B V\}$, $\{B V I\}$, and $\{V I\}$ filter subsets. The ridge lines correspond to unweighted leastsquares fits to the derived EPM quantities. The upper and lower panels show the results using E96 and D05 dilution factors, respectively. In all cases we employ the DES reddening.

(A color version of this figure is available in the online journal.)

from the EPM solution because it shows a clear departure from the linear $\theta / v$ versus $t$ relation. The EPM solutions using the E96 and D05 models are quite linear and show great detail in the evolution of $\theta / v$ due to the high-quality spectroscopic and photometric coverage. However, the E96 solution shows a small departure from linearity in the last two spectroscopic epochs. This effect is probably due to the high rise in the $V_{H \beta} / V_{\text {phot }}$ ratio at low velocities in the E96 models.

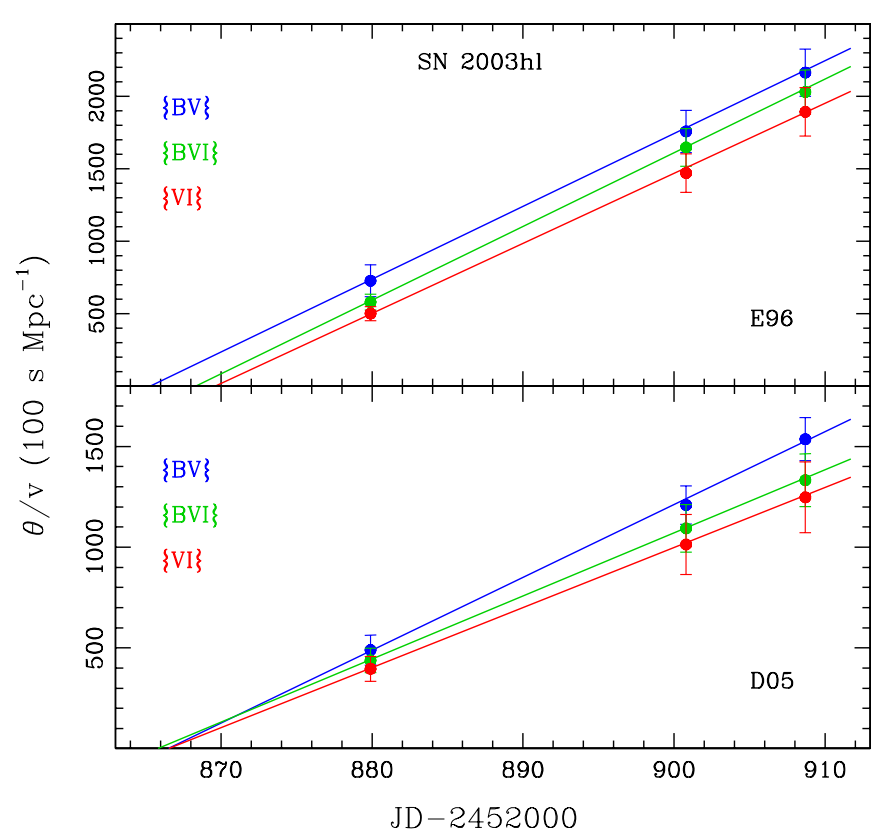

Figure 21. The ratio $\theta / v$ as a function of time for SN 2003hl using the $\{B V\}$, $\{B V I\}$, and $\{V I\}$ filter subsets. The ridge lines correspond to unweighted leastsquares fits to the derived EPM quantities. The upper and lower panels show the results using E96 and D05 dilution factors, respectively. In all cases we employ the DES reddening.

(A color version of this figure is available in the online journal.)

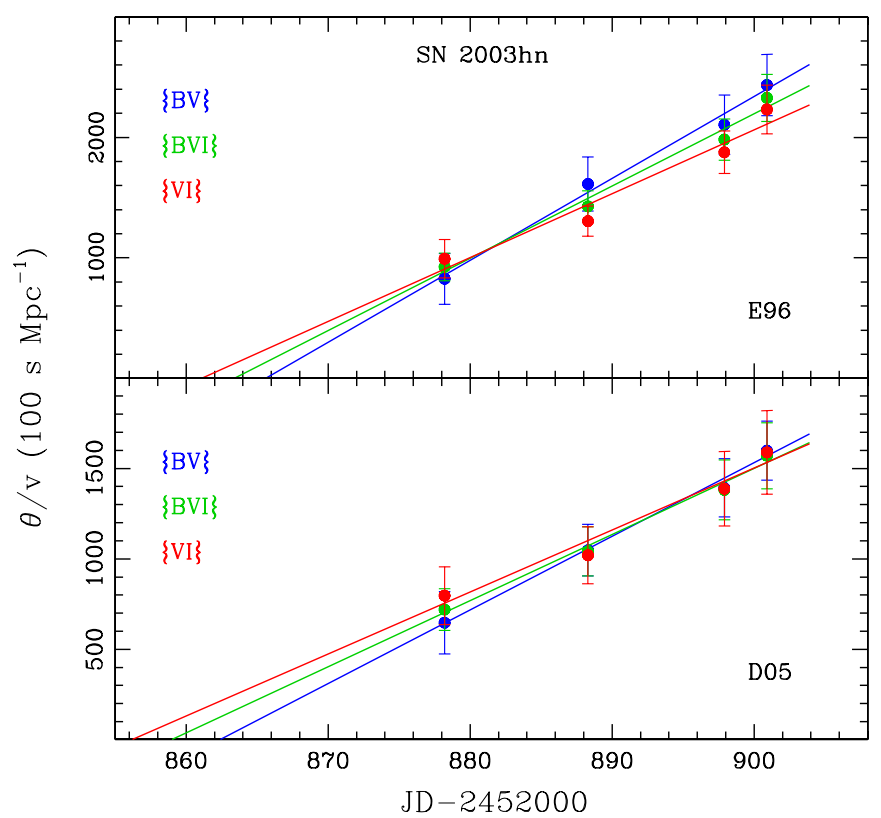

Figure 22. The ratio $\theta / v$ as a function of time for SN 2003hn using the $\{B V\}$, $\{B V I\}$, and $\{V I\}$ filter subsets. The ridge lines correspond to unweighted leastsquares fits to the derived EPM quantities. The upper and lower panels show the results using E96 and D05 dilution factors, respectively. In all cases we employ the DES reddening.

(A color version of this figure is available in the online journal.)

SN 1999em was discovered on JD 2451480.9 by the LOSS (Li 1999b). An image taken at the position of the SN on JD 2451472.0 showed nothing at a limiting magnitude of 19.0. The EPM yields $t_{0}=\mathrm{JD} 2451476.3 \pm 1.1$ and $t_{0}=\mathrm{JD} 2451474.0$ \pm 2.0 using the E96 and D05 models, respectively. These explosions dates are between the pre-discovery and discovery dates. The distances derived to $\mathrm{SN} 1999 \mathrm{em}$ are $D=9.3 \pm$ $0.5 \mathrm{Mpc}$ from E96 and $D=13.9 \pm 1.4 \mathrm{Mpc}$ from D05. 


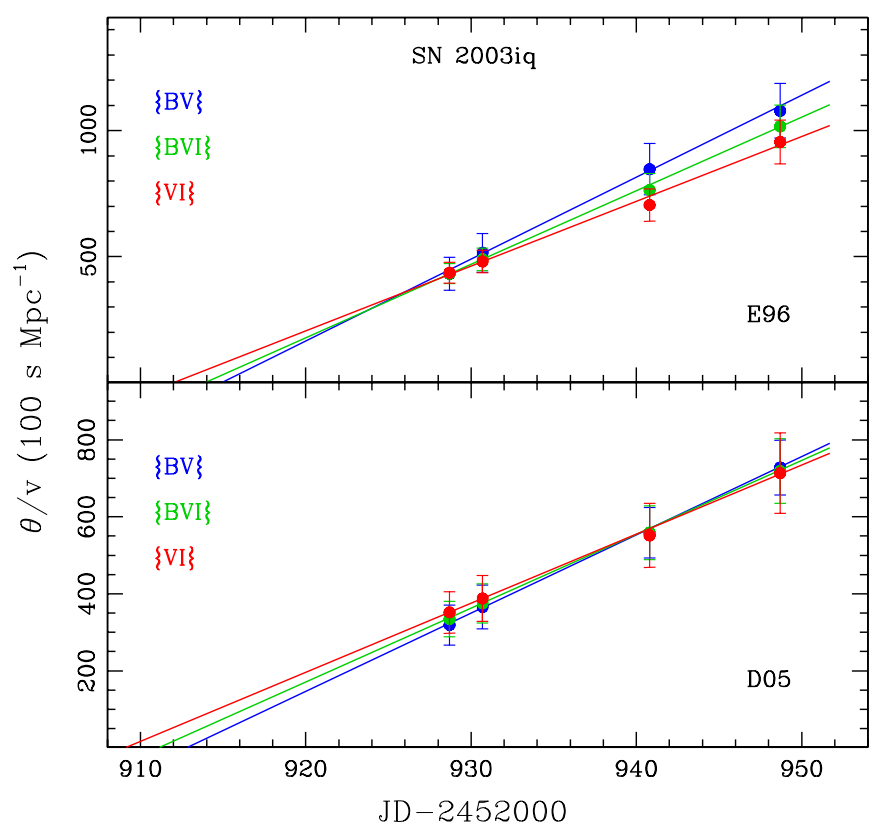

Figure 23. The ratio $\theta / v$ as a function of time for SN 2003iq using the $\{B V\}$, $\{B V I\}$, and $\{V I\}$ filter subsets. The ridge lines correspond to unweighted leastsquares fits to the derived EPM quantities. The upper and lower panels show the results using E96 and D05 dilution factors, respectively. In all cases we employ the DES reddening.

(A color version of this figure is available in the online journal.)

Table 5

EPM Distances

\begin{tabular}{lcccc}
\hline \hline SN & $\begin{array}{c}D_{(E 96)} \\
(\mathrm{Mpc})\end{array}$ & $\begin{array}{c}t_{0(E 96)} \\
(\mathrm{JD}-2448000)\end{array}$ & $\begin{array}{c}D_{(D 05)} \\
(\mathrm{Mpc})\end{array}$ & $\begin{array}{c}t_{0(D 05)} \\
(\mathrm{JD}-2448000)\end{array}$ \\
\hline $1992 \mathrm{ba}$ & $16.4(2.5)$ & $883.9(3.0)$ & $27.2(6.5)$ & $879.8(5.6)$ \\
$1999 \mathrm{br}$ & $\ldots$ & $\ldots$ & $39.5(13.5)$ & $3275.6(7.7)$ \\
$1999 \mathrm{em}$ & $9.3(0.5)$ & $3476.3(1.1)$ & $13.9(1.4)$ & $3474.0(2.0)$ \\
$1999 \mathrm{gi}$ & $11.7(0.8)$ & $3517.0(1.2)$ & $17.4(2.3)$ & $3515.6(2.4)$ \\
$2002 \mathrm{gw}$ & $37.4(4.9)$ & $4557.9(2.7)$ & $63.9(17.0)$ & $4551.7(7.6)$ \\
$2003 \mathrm{~T}$ & $87.8(13.5)$ & $4654.2(2.7)$ & $147.3(35.7)$ & $4648.9(6.1)$ \\
$2003 \mathrm{bl}$ & $\ldots$ & $\ldots$ & $92.4(14.2)$ & $4694.5(2.0)$ \\
$2003 \mathrm{bn}$ & $50.2(7.0)$ & $4693.4(2.7)$ & $87.2(28.0)$ & $4687.0(9.0)$ \\
$2003 \mathrm{ef}$ & $38.7(6.5)$ & $4759.8(4.7)$ & $74.4(30.3)$ & $4748.4(15.6)$ \\
$2003 \mathrm{hl}$ & $17.7(2.1)$ & $4872.3(1.7)$ & $30.3(6.3)$ & $4865.4(5.9)$ \\
$2003 \mathrm{hn}$ & $16.9(2.2)$ & $4859.5(3.8)$ & $26.3(7.1)$ & $4853.8(9.3)$ \\
$2003 \mathrm{iq}$ & $36.0(5.6)$ & $4909.6(4.3)$ & $53.3(17.1)$ & $4905.6(9.5)$ \\
\hline
\end{tabular}

Note. The distances were derived using the $\{V I\}$ filter subset and DES reddening.

$S N$ 1999gi. Figure 15 shows $\theta / v$ versus time for SN 1999gi using the $\{B V\},\{B V I\}$, and $\{V I\}$ filter subsets and the E96 and D05 models. We used five epochs (JD 2451525.0-2451556.9) to apply the EPM method. All of the spectra and the photometry were taken from Leonard et al. (2002a). The first spectrum (JD 2451522.9) was removed from the EPM solutions because it yields an $\mathrm{H} \beta$ velocity of $\sim 26,000 \mathrm{~km} \mathrm{~s}^{-1}$, well above the range of the photospheric velocity conversion (see Section 3.4 and Figure 8). The explosion dates of SN 1999gi obtained using the EPM are $t_{0}=\mathrm{JD} 2451517.0 \pm 1.2$ using the E96 models and $t_{0}=\mathrm{JD} 2451515.6 \pm 2.4$ with D05. These results agree with the observations because a pre-discovery image taken on JD 2451515.7 (Trondal et al. 1999) showed nothing at the SN position (limiting unfiltered magnitude of 18.5). SN 1999gi was discovered on JD 2451522.3 (Nakano et al. 2002) on unfiltered $\mathrm{CCD}$ frames, so the explosion date can be constrained in a
Table 6

Error Sources

\begin{tabular}{lc}
\hline \hline Error Source & Typical Error \\
\hline Photometry & $0.02 \mathrm{mag}$ \\
SN redshift & $50 / 200\left(\mathrm{~km} \mathrm{~s}^{-1}\right)^{\mathrm{a}}$ \\
Foreground extinction & $0.02 \mathrm{mag}$ \\
Host-galaxy extinction & $0.15 \mathrm{mag}$ \\
Line expansion velocity & $85\left(\mathrm{~km} \mathrm{~s}^{-1}\right)$ \\
Photospheric velocity conversion & $0.06 / 0.04^{\mathrm{b}}$ \\
Dilution Factors & $0.03 / 0.07^{\mathrm{b}}$
\end{tabular}

Notes.

${ }^{\text {a }}$ Corresponds to the redshifts measured in this work and those taken from NED, respectively.

${ }^{\mathrm{b}}$ Corresponds to the E96 and D05 models, respectively.

Table 7

SN 1999em EPM Quantities

\begin{tabular}{lcccrc}
\hline \hline JD- & $\begin{array}{c}T_{V I} \\
2451000\end{array}$ & $\begin{array}{c}\theta \zeta_{V I} \\
\left(10^{15} \mathrm{~cm} \mathrm{Mpc}^{-1}\right)\end{array}$ & $\zeta_{V I}$ & $\begin{array}{c}v_{\text {phot }} \\
\left(\mathrm{km} \mathrm{s}^{-1}\right)\end{array}$ & $\begin{array}{c}\theta / v e l \\
\left(100 \mathrm{spc}^{-1}\right)\end{array}$ \\
\hline 482.8 & $14588(469)$ & $0.0321(0.0010)$ & 0.574 & 11022 & $506.7(73.0)$ \\
483.8 & $14349(462)$ & $0.0331(0.0011)$ & 0.572 & 10355 & $559.6(81.0)$ \\
484.8 & $13986(382)$ & $0.0341(0.0009)$ & 0.568 & 9867 & $608.3(88.1)$ \\
485.2 & $13810(415)$ & $0.0345(0.0011)$ & 0.566 & 9117 & $669.5(97.7)$ \\
485.7 & $13550(414)$ & $0.0352(0.0011)$ & 0.563 & 8942 & $699.7(102.6)$ \\
485.7 & $13544(414)$ & $0.0352(0.0011)$ & 0.563 & 8915 & $702.1(103.0)$ \\
485.8 & $13479(456)$ & $0.0353(0.0012)$ & 0.562 & 9311 & $675.0(99.7)$ \\
486.8 & $12812(425)$ & $0.0373(0.0013)$ & 0.555 & 8817 & $762.6(113.9)$ \\
487.9 & $11985(333)$ & $0.0403(0.0013)$ & 0.547 & 8584 & $857.5(128.9)$ \\
488.8 & $11587(310)$ & $0.0413(0.0013)$ & 0.544 & 8598 & $882.8(133.3)$ \\
489.8 & $11352(256)$ & $0.0424(0.0011)$ & 0.542 & 8476 & $921.1(138.7)$ \\
491.1 & $11077(350)$ & $0.0443(0.0016)$ & 0.541 & 7870 & $1040.1(159.5)$ \\
491.2 & $11055(358)$ & $0.0444(0.0017)$ & 0.541 & 7824 & $1050.6(161.4)$ \\
491.7 & $10939(372)$ & $0.0453(0.0018)$ & 0.540 & 7964 & $1053.3(162.6)$ \\
492.1 & $10840(349)$ & $0.0460(0.0018)$ & 0.539 & 7863 & $1083.7(166.9)$ \\
496.2 & $10264(312)$ & $0.0495(0.0019)$ & 0.537 & 7031 & $1311.6(202.6)$ \\
496.7 & $10224(301)$ & $0.0497(0.0018)$ & 0.537 & 7172 & $1290.7(199.0)$ \\
501.2 & $9610(224)$ & $0.0526(0.0016)$ & 0.537 & 5921 & $1653.2(252.9)$ \\
501.7 & $9386(185)$ & $0.0548(0.0014)$ & 0.538 & 6107 & $1667.6(253.4)$ \\
501.7 & $9384(185)$ & $0.0548(0.0014)$ & 0.538 & 6250 & $1630.3(247.7)$ \\
501.8 & $9362(189)$ & $0.0551(0.0015)$ & 0.538 & 6506 & $1572.5(238.9)$ \\
504.8 & $8907(173)$ & $0.0589(0.0016)$ & 0.542 & 5991 & $1813.0(274.0)$ \\
506.8 & $8655(162)$ & $0.0605(0.0016)$ & 0.545 & 5691 & $1950.5(293.2)$ \\
510.8 & $8248(63)$ & $0.0649(0.0007)$ & 0.553 & 5156 & $2276.6(334.0)$ \\
514.8 & $7819(92)$ & $0.0705(0.0013)$ & 0.565 & 4877 & $2557.8(370.3)$ \\
& & & & &
\end{tabular}

Note. The EPM quantities were derived using the $\{V I\}$ filter subset, the DES reddening and the D05 models.

range of only 6.6 days. We derive a distance of $D=11.7 \pm$ $0.8 \mathrm{Mpc}$ and $D=17.4 \pm 2.3 \mathrm{Mpc}$ using the E96 and D05 models, respectively.

$S N$ 2002gw. Figure 16 shows $\theta / v$ versus time for SN 2002gw using the $\{B V\},\{B V I\}$, and $\{V I\}$ filter subsets and the E96 and D05 models. The EPM solutions were obtained using six epochs (JD 2452573.1-2452590.7). The EPM yields explosion times of $t_{0}=\mathrm{JD} 2452557.9 \pm 2.7$ and $t_{0}=\mathrm{JD} 2452551.7 \pm 7.6$ (using E96 and D05 dilution factors, respectively). SN 2002gw was discovered on JD 2452560.8 (Monard 2002). An image taken on JD 2452529.6 shows nothing at the SN position at a limiting magnitude of 18.5. Also, an unfiltered CCD image taken on JD 2452559.1 shows the SN at magnitude 18.3 (Itagaki \& Nakano 2002). The EPM explosion dates are in agreement with the SN explosion date constrained by the observations. The EPM distances are $D=37.4 \pm 4.9 \mathrm{Mpc}$ and $D=63.9 \pm$ 17.0 Mpc using E96 and D05, respectively. 
$S N 2003 T$. Figure 17 shows $\theta / v$ versus time for SN 2003T using the $\{B V\},\{B V I\}$, and $\{V I\}$ filter subsets and the E96 and D05 models. The EPM solutions were obtained using three epochs (JD 2452667.9-2452701.7). The EPM explosion dates are $t_{0}=$ JD $2452654.2 \pm$ using E96 models and $t_{0}=$ JD $2452648.9 \pm$ 3.4 with D05. In both cases the third epoch used to derive the distance is beyond $\sim 45$ days after the EPM $t_{0}$, but it proves necessary to include it to compute the EPM analysis. This SN was discovered by the Lick and Tenagra Observatories Supernova Search (LOTOSS) on JD 2452664.9 (Schwartz \& Li 2003). An image taken on JD 2452644.9 shows nothing at a limiting magnitude of 19.0, in good agreement with the EPM analysis. The EPM distances are $D=87.8 \pm 13.5 \mathrm{Mpc}$ using E96 and $D=147.3 \pm 35.7 \mathrm{Mpc}$ with D05.

$S N 2003 b l$. Figure 18 shows $\theta / v$ versus time for $\mathrm{SN} 2003 \mathrm{bl}$ using the $\{B V\},\{B V I\}$, and $\{V I\}$ filter subsets and D05 models. The EPM solutions were obtained using four epochs (JD 2452701.8-2452735.8). As with the SN 1999br, we were unable to apply the EPM using E96 because we only had two spectra with velocities higher than $4500 \mathrm{~km} \mathrm{~s}^{-1}$, and so the photospheric velocity correction could not be applied (see Section 3.4 and Figure 8). SN 2003bl was discovered by LOTOSS on JD 2452701.0 (Swift et al. 2003). A pre-discovery image taken on JD 2452438.8 shows nothing at the SN position at a limiting magnitude of 19.0. The EPM yields $t_{0}=$ JD $2452692.6 \pm 2.8$, consistent with the SN discovery date. The EPM distance is $D=92.4 \pm 14.2 \mathrm{Mpc}$.

$S N 2003 b n$. Figure 19 shows $\theta / v$ versus time for $\mathrm{SN} 2003 \mathrm{bn}$ using the $\{B V\},\{B V I\}$, and $\{V I\}$ filter subsets and the E96 and D05 models. We computed the EPM analysis using three epochs (JD 2452706.6-2452729.7). The EPM yields explosion dates of $t_{0}=\mathrm{JD} 2452693.4 \pm 2.7$ and $t_{0}=\mathrm{JD} 2452687.0 \pm 9.0$ from E96 and D05, respectively. SN 2003bn was discovered on JD 2452698.0 (Wood-Vasey et al. 2003). Two pre-discovery NEAT images show nothing at the SN position on JD 2452691.5 (limiting magnitude of 21.0) and the SN at a magnitude of 20.2 on JD 2452692.8, which restricted the explosion date in a range of only 1.3 days. This value for $t_{0}$ is in agreement within one $\sigma$ with the EPM $t_{0}$ derived using E96 and D05. The EPM distances from E96 and D05 are $D=50.2 \pm 7.0 \mathrm{Mpc}$ and $D=87.2 \pm$ $28.0 \mathrm{Mpc}$, respectively.

$S N$ 2003ef. Figure 20 shows $\theta / v$ versus time for SN 2003ef using the $\{B V\},\{B V I\}$, and $\{V I\}$ filter subsets and the E96 and D05 models. We computed the EPM analysis using four epochs (JD 2452780.7-2452797.6). The explosion dates derived are $t_{0}=\mathrm{JD} 2452759.8 \pm 4.7$ and $t_{0}=\mathrm{JD} 2452748.4 \pm 15.6$ with E96 and D05, respectively. SN 2003ef was discovered by the LOTOSS on JD 2452770.8 (magnitude about 16.3; Weisz \& Li 2003), consistent with the EPM value of $t_{0}$. A KAIT image taken on JD 2452720.8 showed nothing at the SN position at a limiting magnitude of 18.5. The EPM distances are $D=38.7 \pm$ $6.53 \mathrm{Mpc}$ with E96 and $D=74.4 \pm 30.3 \mathrm{Mpc}$ with D05.

SN 2003hl. Figure 21 shows $\theta / v$ versus time for SN $2003 \mathrm{hl}$ using the $\{B V\},\{B V I\}$, and $\{V I\}$ filter subsets and the E96 and D05 models. The EPM solutions were obtained using three epochs (JD 2452879.9-2452908.7). We estimated the explosion dates on $t_{0}=\mathrm{JD} 2452872.3 \pm 1.7$ and $t_{0}=\mathrm{JD} 2452865.4 \pm 5.9$ using E96 and D05, respectively. SN 2003hl was discovered on JD 2452872.0 during the LOTOSS program at a magnitude of 16.5 (Moore et al. 2003). A pre-discovery KAIT image taken on JD 2452863.0 shows nothing at the SN position at a limiting magnitude of 19.0. This image restricts the explosion date in a range of 9 days. The EPM explosion dates are in agreement with the observations (within one $\sigma$ ). We derived EPM distances of $D=17.7 \pm 2.1 \mathrm{Mpc}$ with E96 and $D=30.3 \pm 6.3 \mathrm{Mpc}$ with D05.

SN 2003hn. Figure 22 shows $\theta / v$ versus time for SN 2003hn using the $\{B V\},\{B V I\}$, and $\{V I\}$ filter subsets and the E96 and D05 models. The EPM solutions were obtained using four epochs (JD 2452878.2-2452900.9). The EPM explosion dates derived are $t_{0}=\mathrm{JD} 2452859.5 \pm 3.8$ and $t_{0}=\mathrm{JD} 2452853.8 \pm$ 9.3 using the E96 and D05 dilution factors, respectively. This SN was discovered on JD 2452877.2 at 14.1 mag by Evans (2003). Evans also reported that the SN was not visible at 15.5 mag on JD 2452856.5. This date agrees with the explosion date derived from E96 and is less than $1 \sigma$ lower than that derived from D05. The EPM solutions lead to $D=16.9 \pm 2.2 \mathrm{Mpc}$ and $D=26.3 \pm 7.1 \mathrm{Mpc}$ using E96 and D05 models, respectively.

$S N 2003 i q$. Figure 23 shows $\theta / v$ versus time for SN 2003iq using the $\{B V\},\{B V I\}$, and $\{V I\}$ filter subsets and the E96 and D05 models. The EPM solutions were obtained using four epochs (JD 2452928.7-2452948.7). This SN was discovered by Llapasset (2003) on JD 2452921.5, while monitoring SN 2003hl in the same host galaxy. A pre-discovery image taken on 2452918.5 shows nothing at the SN position. These reports constrain the explosion date to a range of only 3 days. The EPM method yields $t_{0}=\mathrm{JD} 2452909.6 \pm 4.3$ using E96 and $t_{0}=\mathrm{JD} 2452905.6 \pm$ 9.5 using D05. In both cases the explosion date is far earlier than expected because the SN was not present on JD 2452918.5. This implies that the EPM solutions to this SN are not satisfactory. We derived EPM distances of $D=36.0 \pm 5.6 \mathrm{Mpc}$ with $E 96$ and $\mathrm{D}=53.3 \pm 17.1 \mathrm{Mpc}$ with D05.

\section{DISCUSSION}

\subsection{External Comparison}

\subsubsection{Previous EPM distances}

The EPM method has already been applied to SN 1999em by other authors, as follows. (1) Hamuy et al. (2001) employed the E96 dilution factors and eight different filter subsets to perform the EPM analysis to this SN, using a cross-correlation technique to estimate the photospheric velocity and adopting a host-galaxy extinction of $A_{V}=0.18 \mathrm{mag}$. They derived a distance of $6.9 \pm$ $0.1,7.4 \pm 0.1$, and $7.3 \pm 0.1 \mathrm{Mpc}$ from the $\{B V\},\{B V I\}$, and $\{V I\}$ filter subsets, respectively. These values are in agreement with our estimates of $6.9 \pm 0.6,7.5 \pm 0.6$, and $9.3 \pm 0.5 \mathrm{Mpc}$ (from the $\{B V\},\{B V I\}$, and $\{V I\}$ filter subsets, respectively), except in the $\{V I\}$ case. (2) Leonard et al. (2002b) employed the E96 models to derive the distance to SN 1999em. They used four weak unblended spectral features (Fe II $\lambda \lambda 4629$, 5276,5318 , and Sc II $\lambda$ 4670) as the photospheric velocity indicators. They adopted a host-galaxy reddening of $A_{V}=0.31$ mag, the same value predicted by DES. They derived a distance of $7.7 \pm 0.2,8.3 \pm 0.2$ and $8.8 \pm 0.3 \mathrm{Mpc}$ from the $\{B V\}$, $\{B V I\}$, and $\{V I\}$ filter subsets, respectively. These results are in agreement with our E96 distances. (3) Elmhamdi et al. (2003) determined a color temperature by fitting blackbody functions to their observed spectra of SN 1999em. They used the E96 models and adopted a reddening of $A_{V}=0.31$ to derive a distance of 7.8 $\pm 0.3 \mathrm{Mpc}$ to this SN. This result is in agreement with our EPM distance using E96, except in the $\{V I\}$ case. (4) Dessart \& Hillier (2006) applied the EPM method to SN 1999em using E96 and D05. They adopted the SN 1999em DES reddening value of 


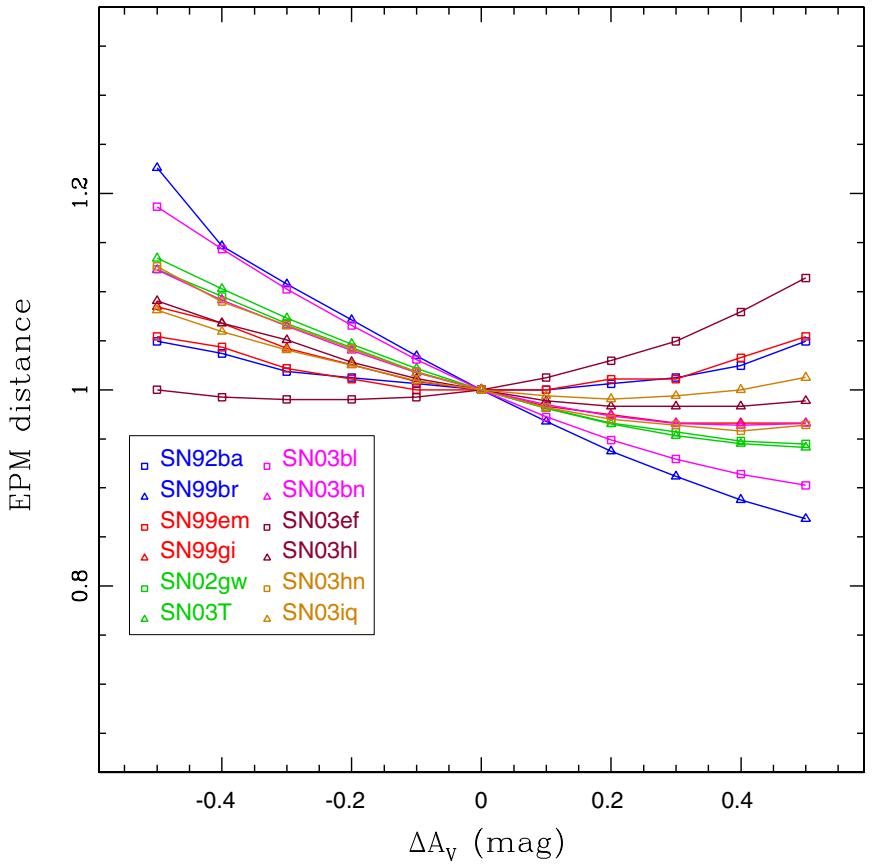

Figure 24. Normalized EPM distances as a function of the host-galaxy visual extinction relative to the DES value $\left(\Delta A_{V}=0\right)$.

(A color version of this figure is available in the online journal.)

$A_{V}=0.31 \mathrm{mag}$. Using the E96 models, they derived a distance of $8.6 \pm 0.8,9.7 \pm 1.0$, and $11.7 \pm 1.5 \mathrm{Mpc}$ from the $\{B V\}$, $\{B V I\}$, and $\{V I\}$ filter subsets, respectively, which are somewhat greater than our distances. They also used specific D05 models for this SN to apply the EPM. They found a distance of $12.4 \pm$ $1.2,12.4 \pm 1.3$, and $12.4 \pm 1.3 \mathrm{Mpc}$ from the $\{B V\},\{B V I\}$, and $\{V I\}$ filter subsets, respectively, using seven epochs and $11.7 \pm$ $1.0,11.6 \pm 1.0$, and $11.5 \pm 0.9 \mathrm{Mpc}$ from the $\{B V\},\{B V I\}$, and $\{V I\}$ filter subsets, respectively, using eight epochs. These results compare very well with our distances using D05 of 11.6 $\pm 1.2,12.1 \pm 0.9$, and $13.9 \pm 1.4 \mathrm{Mpc}$ from the $\{B V\},\{B V I\}$, and $\{V I\}$ filter subsets, respectively.

\subsubsection{SEAM Distance}

SEAM is a technique similar to the EPM, but it avoids the use of dilution factors by doing synthetic spectral fitting to the observed spectra of the SN. Baron et al. (2004) applied this method to SN 1999em. They derived a distance of $D$ $=12.5 \pm 2.3 \mathrm{Mpc}$, in good agreement with our distances derived using the D05 models $(11.6 \pm 1.2,12.1 \pm 0.9$, and $13.9 \pm 1.4 \mathrm{Mpc}$ from the $\{B V\},\{B V I\}$, and $\{V I\}$ filter subsets, respectively), but significantly greater than the EPM distances derived using E96 $(6.9 \pm 0.6,7.5 \pm 0.6$ and $9.3 \pm 0.5 \mathrm{Mpc}$ from the $\{B V\},\{B V I\}$, and $\{V I\}$ filter subsets, respectively). Also Dessart \& Hillier (2006) applied this technique to SN 1999em, deriving a SEAM distance of $\sim 12.2 \mathrm{Mpc}(11.5 \mathrm{Mpc})$ using seven (eight) observations, in agreement with the Baron et al. (2004) results.

\subsubsection{Cepheid Distance}

Leonard et al. (2003) identified 41 Cepheid variable stars in NGC 1637, the host galaxy of SN 1999em. They derived a Cepheid distance to NGC 1637 of $D=11.7 \pm 1.0 \mathrm{Mpc}$. As with the SEAM results, the Cepheid distance is consistent with our EPM distances from the D05 models $(11.2 \pm 0.2,12.0 \pm 0.2$, and
$14.0 \pm 0.2 \mathrm{Mpc}$ from the $\{B V\},\{B V I\}$, and $\{V I\}$ filter subsets, respectively). In all cases, the E96 models lead to significantly lower distances $(6.9 \pm 0.6,7.5 \pm 0.6$, and $9.3 \pm 0.5 \mathrm{Mpc}$ from the $\{B V\},\{B V I\}$, and $\{V I\}$ filter subsets, respectively).

\subsection{Error Analysis \\ 4.2.1. Effects of Reddening}

While the Schlegel et al. (1998) IR maps provide a reasonable estimate of the amount of Galactic foreground extinction, the determination of host-galaxy extinction is a more challenging task. This is a potential problem because the distances derived using EPM depend on the adopted host-galaxy extinction. In order to investigate the sensitivity of the distances to dust extinction, we performed the EPM analysis of all the $\mathrm{SNe}$ in our sample using the $\{V I\}$ filter subset by varying the amount of host-galaxy visual extinction $A_{V}$ in steps of $\Delta A_{V}=0.1 \mathrm{mag}$. Figure 24 shows the normalized EPM distances as a function of host-galaxy visual extinction $A_{V}$ relative to the DES value $\left(\Delta A_{V}=0\right)$. As can be seen, the EPM is quite insensitive to the amount of host-galaxy extinction adopted. On average, the distances change by less than $\sim 10 \%$ from $\Delta A_{V}=0.0$ to $\Delta A_{V}=0.5 \mathrm{mag}$ and by less than $\sim 20 \%$ from $\Delta A_{V}=0.0$ to $\Delta A_{V}=-0.5 \mathrm{mag}$. Therefore, even a large systematic error of $0.5 \mathrm{mag}$ in $A_{V}$ will not translate into a large error in the EPM distance. This effect is discussed in Eastman \& Kirshner (1989).

\subsubsection{Other Sources of Error}

Table 6 lists all the error sources in EPM and their typical values. In order to investigate which source contributes the most to the uncertainty in the EPM distance, we performed the EPM analysis of SN 1999gi (whose photometry and spectroscopy coverage is representative of our sample), and we changed the error of a single source (listed in Table 6) leaving all others unchanged. We found two main sources of error. In the E96 case, the errors in the photospheric velocity conversion and the dilution factors have the largest effect in the distance uncertainty, each one contributing $\sim 30 \%$ of the total error, while in the D05 case the error in the dilution factors produces $\sim 70 \%$ of the uncertainty in the distance, far greater than that due to the error in the photospheric velocity conversion $(\sim 10 \%$ of the total error). All of the other errors have a secondary effect in the total error.

\subsection{Hubble Diagrams}

Since the discovery of the expansion of the Universe (Hubble 1929), the determination of the expansion rate, the Hubble constant $\left(H_{0}\right)$, has become one of the most important challenges in astronomy and cosmology. Using the velocitydistance relation (Hubble diagram) calibrated with the Cepheid period-luminosity relation, Hubble \& Humason (1931) obtained $\mathrm{H}_{0} \sim 500 \mathrm{kms}^{-1} \mathrm{Mpc}^{-1}$. During the second half of the 20th century, the Cepheid relation was significantly improved, and new Hubble diagrams were obtained, yielding Hubble constants in the range $\sim 50-100 \mathrm{~km} \mathrm{~s}^{-1} \mathrm{Mpc}^{-1}$. Today the discrepancy is not over, but there is a convergence into a value of $H_{0} \sim 65-80 \mathrm{~km}$ $\mathrm{s}^{-1} \mathrm{Mpc}^{-1}$ ) (Sandage et al. 2006; Freedman et al. 2001; Riess et al. 2005). In this work we applied the EPM method to 12 SNe using two sets of dilution factors (E96, D05), two extinction determination methods (OLI, DES), and three filter subsets $(\{B V\},\{B V I\}$, and $\{V I\})$ to derive their distances. In order to obtain the host-galaxy redshifts relative to the cosmic microwave background (CMB), we corrected the heliocentric host-galaxy 


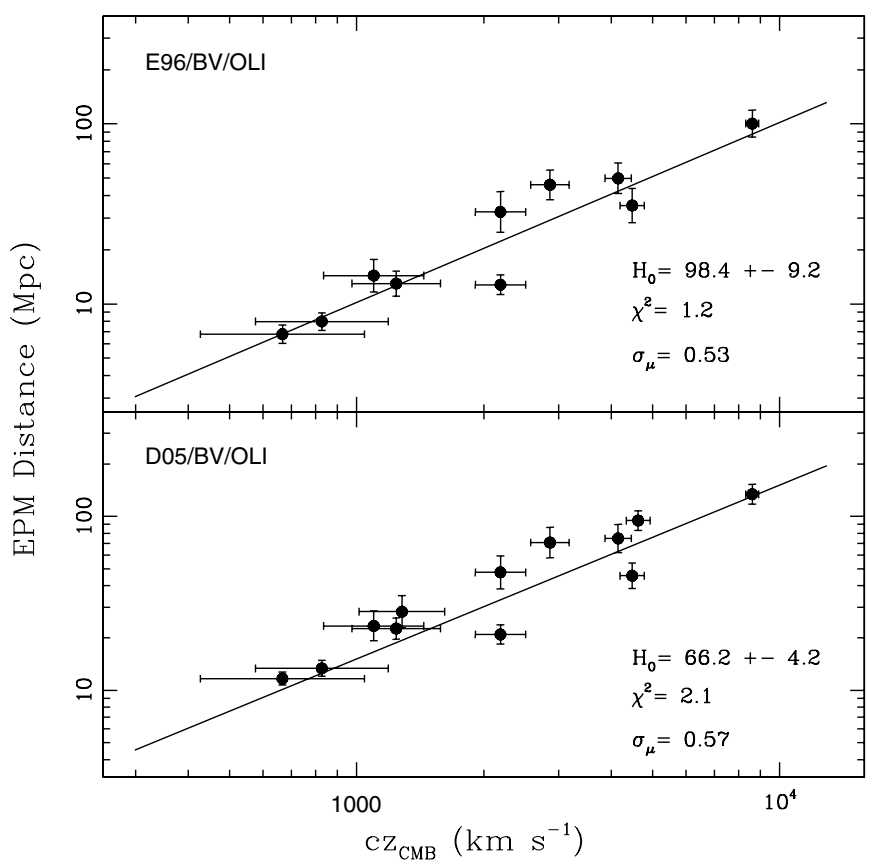

Figure 25. Hubble diagram using the $\{B V\}$ filter subset and OLI reddening.

(A color version of this figure is available in the online journal.)

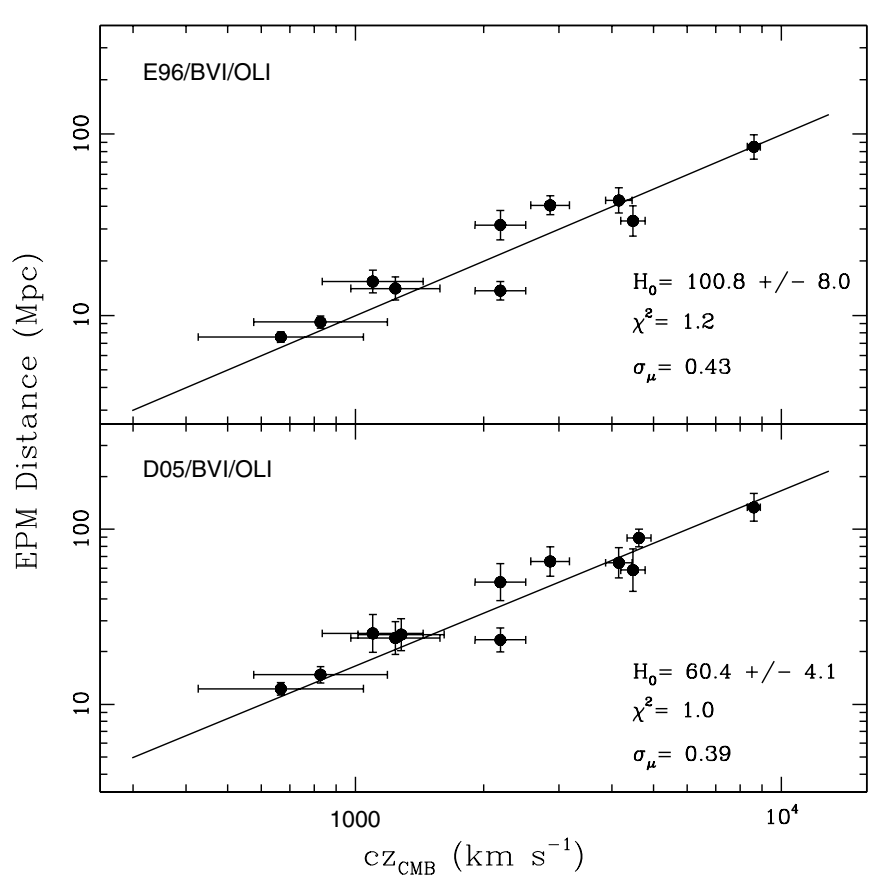

Figure 26. Hubble diagram using the $\{B V I\}$ filter subset and OLI reddening.

redshifts for the peculiar velocity of the Sun relative to the CMB rest frame. For this purpose we added a velocity vector of 371 $\mathrm{km} \mathrm{s}^{-1}$ in the direction $(l, b)=\left(264.14,48^{\circ} .26\right.$; Fixsen et al. 1996 ) to the heliocentric redshifts. The resulting CMB redshifts are given in Table 1.

Using the CMB host-galaxy redshifts, we constructed 12 different Hubble diagrams. In each case we computed a linear fit weighting the error in distance and redshift (assumed to be $300 \mathrm{~km} \mathrm{~s}^{-1}$ for all SNe) in order to derive the Hubble constant. Figures 25-27 show the Hubble diagrams obtained with OLI reddenings, from the $\{B V\},\{B V I\}$ and $\{V I\}$ filter subsets, respectively. Figures $28-30$ show the same diagrams but this

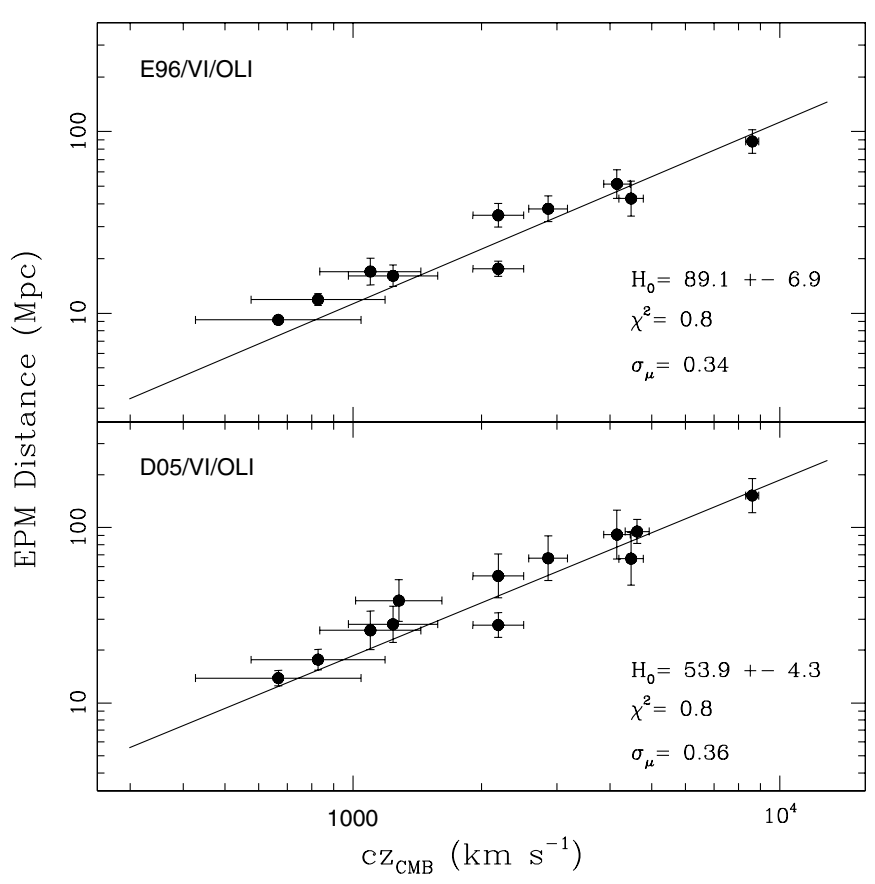

Figure 27. Hubble diagram using the $\{V I\}$ filter subset and OLI reddening.

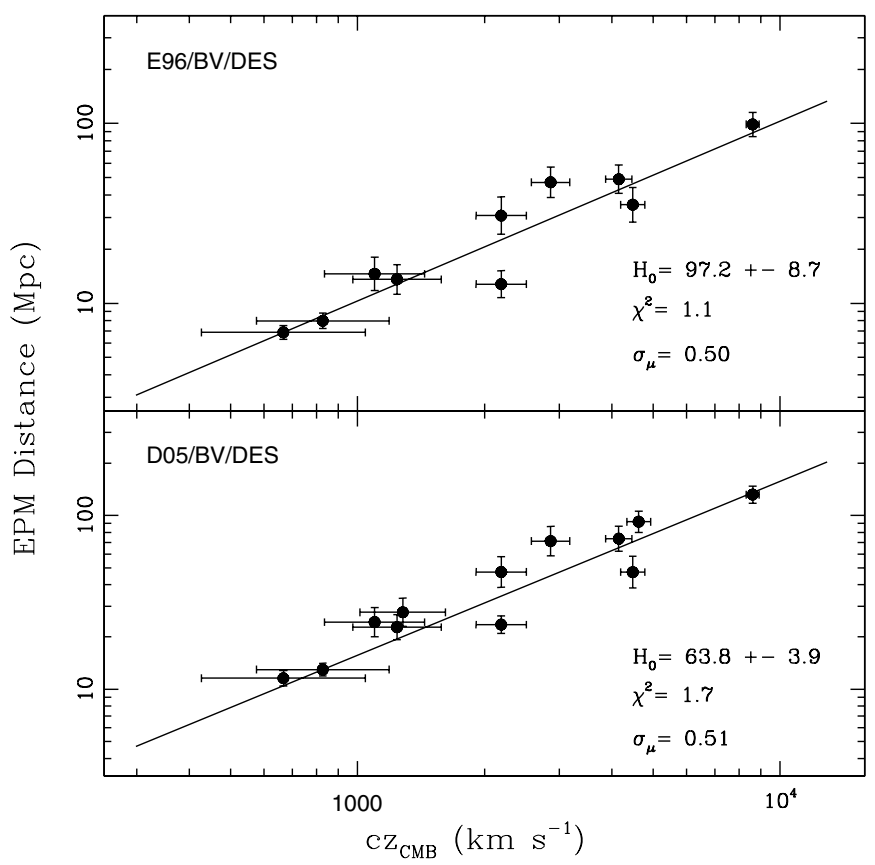

Figure 28. Hubble diagram using the $\{B V\}$ filter subset and DES reddening.

time using DES extinctions. Each diagram is labeled with the derived Hubble constant, the reduced $\chi^{2}$, and the dispersion in distance modulus $\sigma_{\mu}$ from the linear fit. The resulting $H_{0}$ values are summarized in Table 8 . There is a systematic difference in the $H_{0}$ values obtained with the E96 and D05 models. Using E96 we obtained $H_{0}=89-101 \mathrm{~km} \mathrm{~s}^{-1} \mathrm{Mpc}^{-1}$ while D05 yielded $H_{0}=52-66 \mathrm{~km} \mathrm{~s}^{-1} \mathrm{Mpc}^{-1}$. This difference arises both from the systematically higher D05 dilution factors and the different photospheric velocity conversion between both models. These two effects combined lead to differences of $\sim 40 \%$ in the EPM distances. The use of different filter subsets leads to $H_{0}$ values consistent within $1 \sigma$ for a fixed atmosphere model. This is a very important result, because it suggests an internal consistency for 


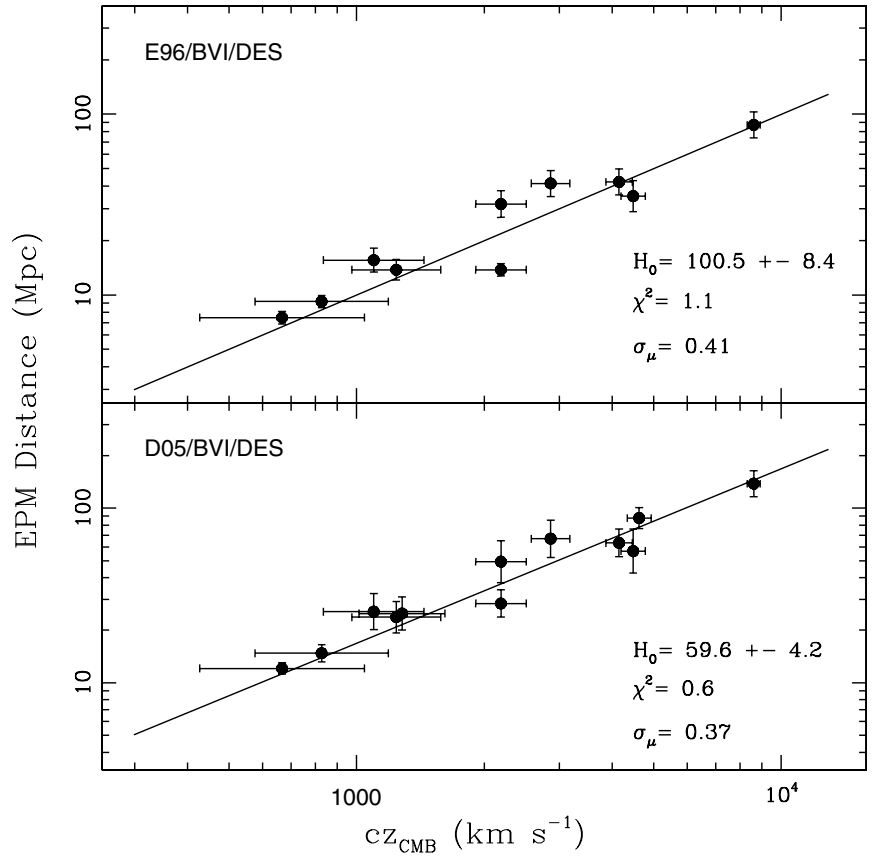

Figure 29. Hubble diagram using the $\{B V I\}$ filter subset and DES reddening.

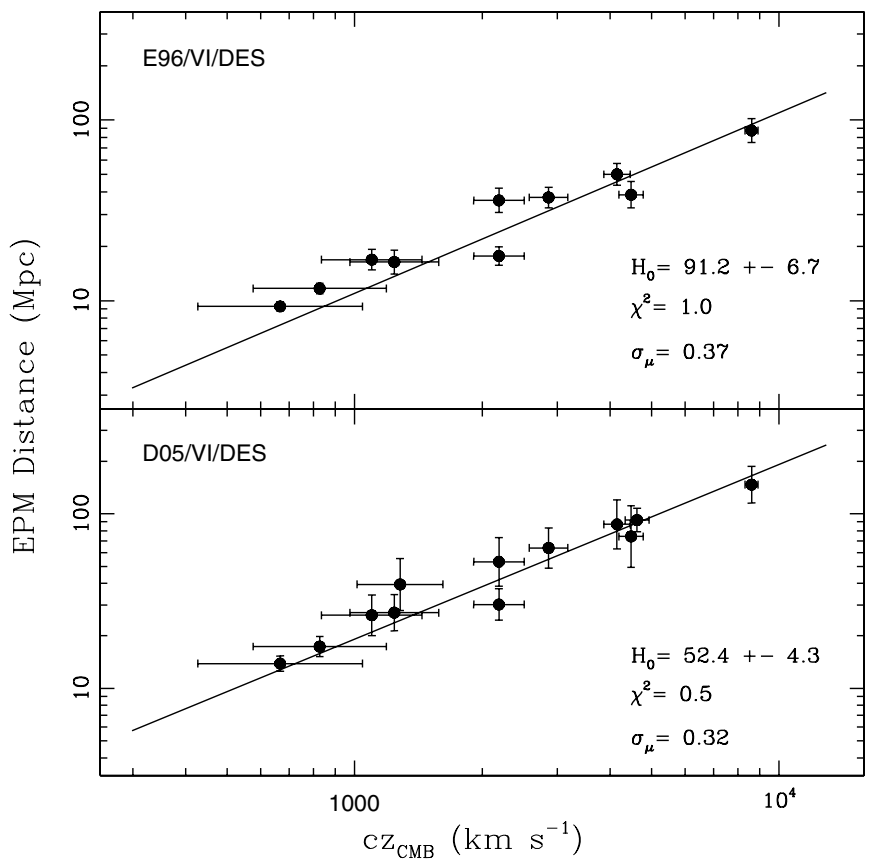

Figure 30. Hubble diagram using the $\{V I\}$ filter subset and DES reddening.

each set of atmosphere models. However, the use of different filter subsets produces significant differences in dispersion, increasing from $\sigma_{\mu} \sim 0.3(V I)$ to $\sigma_{\mu} \sim 0.4(B V I)$ and $\sigma_{\mu} \sim 0.5$ $(B V)$ (see Table 9). The special case of D05 with VI and DES, leads to $\sigma_{\mu}=0.32$, which corresponds to $\sim 15 \%$ of error in distance. Clearly when the $B$ band is employed, the dispersion in the Hubble diagram increases considerably. This is due to the fact that the dilution factors in the $B$ band, are mostly determined by the effect of line blanketing rather than by electron scattering, so the assumption of a "dilute" blackbody becomes less reliable. It is also possible that metallicity differences of the SNe could explain part of this scatter, although both atmosphere model sets predict a modest effect of metallicity in the emergent
Table 8

Summary of $\mathrm{H}_{0}$ Values

\begin{tabular}{lccc}
\hline \hline & $\{B V\}$ & $\{B V I\}$ & $\{V I\}$ \\
\hline E96/OLI & $98.4(9.2)$ & $100.8(8.0)$ & $89.1(6.9)$ \\
E96/DES & $97.2(8.7)$ & $100.5(8.4)$ & $91.2(6.7)$ \\
D05/OLI & $66.2(4.2)$ & $60.4(4.1)$ & $53.9(4.3)$ \\
D05/DES & $63.8(3.9)$ & $59.6(4.2)$ & $52.4(4.3)$ \\
\hline
\end{tabular}

Table 9

Summary of Dispersions in Hubble Diagrams

\begin{tabular}{lccc}
\hline \hline & $\{B V\}$ & $\{B V I\}$ & $\{V I\}$ \\
\hline E96/OLI & 0.53 & 0.43 & 0.34 \\
E96/DES & 0.50 & 0.41 & 0.37 \\
D05/OLI & 0.57 & 0.39 & 0.36 \\
D05/DES & 0.51 & 0.37 & 0.32 \\
\hline
\end{tabular}

flux at wavelengths longer than $\sim 4000 \AA$. As expected, it can be noted that there are no significant differences in the $H_{0}$ values and in the Hubble diagram dispersion between the DES and OLI reddening methods. This is because there is no systematic difference in the reddening between both methods (see Section 3.5). However, the DES method leads to somewhat lower dispersion in the Hubble diagrams than the OLI technique. Finally, SN 2003hl and SN 2003iq are of particular interest because they both exploded in the same galaxy. To our disappointment, all 12 possible combinations of filter subsets, reddening, and atmosphere models lead to significant differences in the EPM distance to the host galaxy. The most extreme case is the $\{B V\}, \mathrm{E} 96$, and OLI combination, which leads to a distance of $32.5 \pm 8.5 \mathrm{Mpc}$ to SN 2003iq and $12.8 \pm 1.6 \mathrm{Mpc}$ to SN 2003hl (a difference of 2.3 sigma). The smallest discrepancy occurs with the $\{V I\}$, D05, and DES combinations $(30.3 \pm 6.3$ and $53.3 \pm 17.1 \mathrm{Mpc}$ for SN 2003hl and SN 2003iq, respectively), which is also the combination that produces the lowest dispersion in the Hubble diagram. As discussed in Section 3, the EPM solutions to SN 2003iq yield an explosion time inconsistent with a pre-discovery image, hence the EPM distance to SN 2003iq is quite suspicious. The light curve and the spectroscopic velocity evolution of both $\mathrm{SNe}$ are typical of the Type IIP class, so this is not the reason for the inconsistency in the EPM solutions. Given the high host-galaxy extinction to SN 2003hl, it is conceivable that the discrepancy could be due to a departure from the standard reddening law. We explored this possibility by varying $R_{V}$ over the range $1.1-$ 3.1 using the $\{V I\}$ and D05 models. For the case $R_{V}=3.1$ we obtain $\mathrm{D}(03 \mathrm{hl}) / \mathrm{D}(03 \mathrm{iq})=0.57$, while for $R_{V}=1.1$ we obtain $\mathrm{D}(03 \mathrm{hl}) / \mathrm{D}(03 \mathrm{iq})=0.72$. This exercise shows that, although the discrepancy is not completely removed, lowering $R_{V}$ brings the two distances in better agreement. Interestingly, Olivares (2008) and Poznanski et al. (2009) found a value of $R_{V}<2$ using the SCM. All this suggests that either (1) the dust around SNe IIP has different properties than the Galactic dust, or (2) SNe IIP are surrounded by Galactic-like dust whose geometric distribution is responsible for an abnormal low value of $R_{V}$ (Wang 2005; Goobar 2008).

\subsection{External Calibration and the Internal precision of the $E P M$}

In the previous section we have shown that there is a systematic difference in the $H_{0}$ values derived using the E96 and the D05 models. In order to remove this systematic effect, 


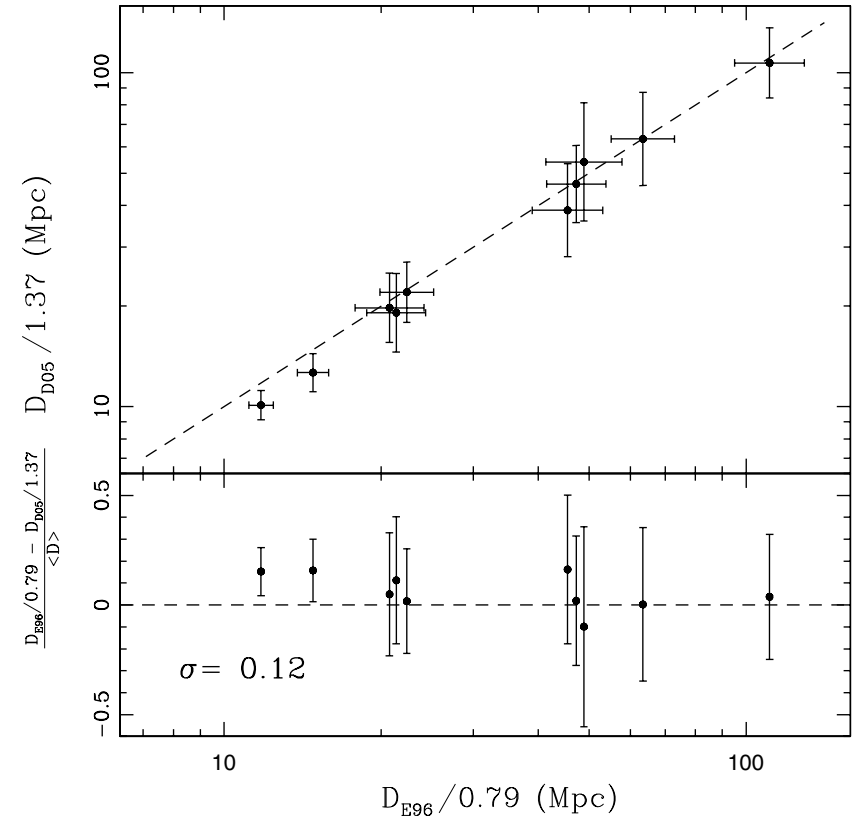

Figure 31. Top panel: D05 distances versus E96 distances corrected to the HST Key Project Cepheid scale. The dashed line shows slope unity. Bottom panel: differences between the corrected distances normalized to the average of the E96 and D05 corrected distances. The $12 \%$ scatter reflects the internal precision of the EPM.

we applied a calibration factor (given by the ratio between some external $H_{0}$ value and the EPM $H_{0}$ value) to the distances derived using E96 and D05. For this purpose we used the value of $\mathrm{H}_{0}=72 \mathrm{~km} \mathrm{~s}^{-1} \mathrm{Mpc}^{-1}$ derived from the Hubble Space Telescope Key Project (Freedman et al. 2001). This external calibration allows us to bring the EPM distances to the Cepheid scale and allows us to remove the systematic difference in the EPM distances between E96 and D05. Figure 31 shows (top panel) the D05 distances versus the E96 distances divided by a calibration factor of 1.37 and 0.79 , respectively. In both cases the EPM distances were derived using the $\{V I\}$ filter subset and the DES reddening. As can be seen, after applying this correction, the systematic differences disappear. The dashed line in the top panel corresponds to the one-to-one relation. Also, in Figure 31 (bottom panel) are plotted the differences between the corrected E96 and D05 distances, normalized to the corresponding average between the corrected E96 and D05 distances. We found a standard deviation of $\sigma=0.12$. Since the dispersion arises from the combined errors in the E96 and the D05 distances, the internal random errors in any of the EPM implementation must be less than $12 \%$. Note that this scatter is smaller than the $\sim 15 \%$ dispersion seen in the Hubble diagrams, which is affected by the peculiar motion of the host galaxies. The $12 \%$ scatter is independent of the redshift and must be an upper value of the internal precision of the EPM.

\section{CONCLUSIONS}

In this work we have applied the EPM method to $12 \mathrm{SNe}$ IIP. We constructed 12 different Hubble diagrams, using three different filter subsets $(\{B V\},\{B V I\},\{V I\})$, two atmosphere models (E96, D05), and two methods to determine the amount of host-galaxy extinction (DES, OLI). Our main conclusions are as follows.

The EPM must be restricted to the first $\sim 45-50$ days from explosion. After that epoch the method may display departures from linearity in the $\theta / v$ versus time relation, and therefore an internal inconsistency.

The results are less precise when the $B$ band is used in the EPM analysis, regardless of the atmosphere models employed (E96 or D05). The dispersion in the Hubble diagrams increases considerably from 0.3 to $0.5 \mathrm{mag}$ when the $B$ band is included and the $V$ band is removed from the filter subset. Despite the loss in precision, there are no significant differences in the resulting distances.

We investigated the effect of host-galaxy reddening in the EPM distances. For this purpose we computed many EPM solutions varying the amount of visual extinction, and we found that a difference of $\Delta A_{V}=0.5 \mathrm{mag}$ leads on average to a difference of $\sim 5 \%-10 \%$ in distance. Therefore, we conclude that the method is quite insensitive to the effect of dust.

Systematic differences in the atmosphere models lead to $\sim 50 \%$ differences in the EPM distances and to values of $H_{0}$ between 52 and $101 \mathrm{~km} \mathrm{~s}^{-1} \mathrm{Mpc}^{-1}$. This effect is due to the systematic difference in the photospheric velocity conversion and the dilution factors. The latter is currently the greatest source of uncertainty in the EPM method. The Hubble diagram with the lowest dispersion $\left(\sigma_{\mu}=0.32 \mathrm{mag}\right)$ was obtained using the combination D05, $\{V I\}$, DES. Despite the systematic uncertainties in the EPM, this dispersion is quite low and corresponds to a precision of $\sim 15 \%$ in distance. This precision is similar to that of the SCM method for type II SNe (Hamuy \& Pinto 2002; Olivares 2008; Poznanski et al. 2009) and to the Tully-Fisher relation for spiral galaxies with a dispersion of $\sigma \sim 0.30$ mag (Sakai et al. 2000). However, the EPM dispersion is greater than that of the $\mathrm{M} / \Delta m_{15}$ relation for Type Ia SNe, which has a dispersion of $\sigma \sim 0.15-0.20 \mathrm{mag}$; however, if the EPM is applied to a sample of SNe IIP in the Hubble flow, the dispersion in the Hubble diagram might decrease. Finally, despite the systematic differences in the $H_{0}$ value, EPM has great potential as an extragalactic distance indicator and can potentially be applied to a sample of high-redshift SNe IIP in order to check in an independent way the accelerating expansion of the universe.

We thank Luc Dessart and Ronald Eastman for providing us with their atmosphere models. We are also grateful to Robert Kirshner for providing some spectra used in this work. We acknowledge support from the Millennium Center for Supernova Science through grant P06-045-F funded by "Programa Bicentenario de Ciencia y Tecnología de CONICYT" and "Programa Iniciativa Científica Milenio de MIDEPLAN." Additional support was provided by CONICYT through Centro de Astrofísica FONDAP (grant 15010003), Center of Excellence in Astrophysics and Associated Technologies (grant PFB 06), and Fondecyt (grant 1060808). A.V.F.'s supernova group at UC Berkeley and KAIT at Lick Observatory are supported by NSF grant AST-06074845, as well as by generous gifts from the Sylvia and Jim Katzman Foundation and the TABASGO Foundation.

\section{REFERENCES}

Baron, E., et al. 2004, ApJ, 616, L91

Cardelli, J. A., Clayton, C., \& Mathis, J. S. 1989, ApJ, 345, 245

Cousins, A. W. J. 1971, R. Obs. Ann. No 7, 86

Dessart, L., \& Hillier, D. J. 2005a, A\&A, 437, 667

Dessart, L., \& Hillier, D. J. 2005b, A\&A, 439, 671

Dessart, L., \& Hillier, D. J. 2006, A\&A, 447, 691

Dessart, L., \& Hillier, D. J. 2008, MNRAS, 383, 57

Dessart, L., et al. 2008, ApJ, 675, 644

Eastman, R. G., \& Kirshner, R. P. 1989, ApJ, 347, 771 
Eastman, R. G., Schmidt, B. P., \& Kirshner, R. 1996, ApJ, 466, 911

Elmhamdi, A., et al. 2003, MNRAS, 338, 939

Evans, R. 1992, IAU Circ., 5625

Evans, R. 2003, IAU Circ., 8116

Filippenko, A. V. 1982, PASP, 94, 715

Filippenko, A. V. 1991a, in SN 1987A and Other Supernovae, ed. I. J. Danziger, K. Kjär (Garching: ESO), 343

Filippenko, A. V. 1991b, in Supernovae, ed. S. E. Woosley (New York: SpringerVerlag), 467

Filippenko, A. V. 1997, ARA\&A, 35, 309

Filippenko, et al. 2001, in ASP Conf. Ser. 246, The Lick Observatory Supernova Search with the Katzman Automatic Imaging Telescope, ed. B. Paczynski, W.-P. Chen, \& C. Lemme (San Francisco, CA: ASP), 121

Fixsen, D. J., et al. 1996, ApJ, 473, 576

Freedman, W. L. 2001, ApJ, 553, 47

Goobar, A. 2008, ApJ, 686, 103

Hamuy, M., \& Pinto, P. A. 2002, ApJ, 566, L63

Hamuy, M., et al. 1992, PASP, 104, 533

Hamuy, M., et al. 1994, PASP, 106, 566

Hamuy, M., et al. 2001, ApJ, 558, 615

Hubble, E. 1929, PNAS, 15, 168

Hubble, E., \& Humason, M. L. 1931, ApJ, 74, 43

Itagaki, K., \& Nakano, S. 2002, IAU Circ., 7996

Johnson, H. L., et al. 1966, Commun. Lunar Plan. Lab., 4, 99

Jones, M. I. 2008, MSc Thesis, Univ. de Chile (arXiv:0810.5538)

King, J. Y. 1999, IAU Circ., 714

Kirshner, R. P., \& Kwan, J. 1974, ApJ, 193, 27

Leonard, D. C., Filippenko, A. V., \& Ardila, D. R. 2001, ApJ, 553, 861
Leonard, D. C., et al. 2002a, AJ, 124, 2490

Leonard, D. C., et al. 2002b, PASP, 114, 35

Leonard, D. C., et al. 2003, ApJ, 594, 247

Li, W. 1999a, IAU Circ., 7143

Li, W. 1999b, IAU Circ., 7294

Llapasset, J-M. 2003, IAU Circ., 8219

Mc Naught, R. H. 1992, IAU Circ., 5632

Monard, L. 2002, IAU Circ., 7995

Moore, M., Li, W., \& Boles, T. IAU Circ., 8184

Nadyoshin, D. K. 2003, MNRAS, 346, 97

Nakano, S., \& Sumoto Kushida, R. 1999, IAU Circ., 7329

Nugent, P., et al. 2006, ApJ, 645, 841

Olivares, F. 2008, MSc Thesis, Univ. de Chile (arXiv:0810.5518)

Patat, F., et al. 1994, A\&A, 282, 731

Poznanski, D., et al. 2009, ApJ, 694, 1067

Riess, A. G., et al. 2005, ApJ, 627, 579

Sakai, S., et al. 2000, ApJ, 529, 698

Sandage, A., et al. 2006, ApJ, 653, 843

Schlegel, E. M. 1990, MNRAS, 244, 269

Schlegel, D. J., Finkbeiner, D. P., \& Davis, M. 1998, ApJ, 500, 525

Schmidt, B., Kirshner, R., \& Eastman, R. 1992, ApJ, 395, 366

Schwartz, M., \& Li, W. 2003, IAU Circ., 8058

Swift, B., Weisz, D., \& Li, W. 2003, IAU Circ., 8086

Trondal, O., et al. 1999, IAU Circ., 7334

Utrobin, V. P. 2007, A\&A, 461, 233

Wang, L. 2005, ApJ, 635, 33

Weisz, D., \& Li, W. 2003, IAU Circ., 8131

Wood-Vasey, W. M., Aldering, G., \& Nugent, P. 2003, IAU Circ., 8088 